\title{
Mitigating Wake Turbulence Risk During Final Approach via Plate Lines
}

\author{
Frank Holzäpfel*, Anton Stephan, Grigory Rotshteyn, Stephan Körner, \\ Norman Wildmann, Lothar Oswald, Thomas Gerz \\ Institut für Physik der Atmosphäre, Deutsches Zentrum für Luft- und Raumfahrt, \\ 82234 Oberpfaffenhofen, Germany \\ Günther Borek, Alexander Floh, Christian Kern, Markus Kerschbaum, \\ Roman Nossal, Johannes Schwarzenbacher, Martin Stieber, Martin Strobel \\ Austro Control GmbH, 1220 Vienna, Austria \\ Lukas Strauss, Clemens Weiß \\ MeteoServe Wetterdienst GmbH / Austro Control GmbH, 1220 Vienna, Austria \\ Sebastian Kauczok, Christian Schiefer \\ LEONARDO Germany GmbH, 41470 Neuss, Germany \\ Harald Czekala, Gerrit Maschwitz \\ RPG Radiometer Physics GmbH, 53340 Meckenheim, Germany \\ and \\ Igor Smalikho \\ V.E. Zuev Institute of Atmospheric Optics SB RAS, Tomsk 634055, Russia
}

\begin{abstract}
To mitigate the risk of wake vortex encounters during final approach, so-called plate lines have been developed. Wake vortices generated by landing aircraft induce secondary vortices at the plates' surfaces that approach the primary vortices and trigger premature wake vortex decay. Each plate line consists of several upright plates that are installed underneath the approach glide path. While the plate line extends perpendicular to the flight direction, its individual plates are oriented in parallel to the runway centerline. In order to obtain the approval of the authorities for the installation of the plate lines at runway 16 of Vienna International Airport, the plate design had to comply with airport requirements like obstacle clearance, stability, and frangibility. During a six-month campaign wake vortex behavior of about 9,500 landings with and without plates was measured simultaneously by three lidars complemented by a comprehensive suite of meteorological instrumentation. The analysis of over 1000 measured wake vortex evolutions indicates that the plate lines reduce the lifetime of long-lived vortices in a safety corridor along the final approach by $21 \%$ to $35 \%$ depending on the aircraft type. This corresponds to a reduction of vortex circulation by about $50 \%$ for the most relevant ICAO separation (Medium behind Heavy).
\end{abstract}

\section{Introduction}

A $\mathrm{S}$ an unavoidable consequence of lift, every flying vehicle generates counter-rotating regions of turbulence known as wake vortices ${ }^{1}$. Wake vortices represent a potential threat to following air traffic. Aircraft must therefore keep to a predetermined minimum separation distance. These aircraft separations limit the capacity of congested airports in a rapidly growing aeronautical environment. The most likely economic scenario for the future European airport demand indicates that there will be around 1.5 million unaccommodated flights in 2040,

\footnotetext{
*Senior Scientist, frank.holzaepfel@dlr.de, Associate Fellow AIAA
} 
constituting approximately $8 \%$ of the expected demand ${ }^{2}$. Such a lack of capacity could mean 160 million passengers would not be able to travel with a potential economic loss of around $€ 88$ billion to the European economy. To date there is no estimate available to what extent this outlook may have to be adjusted in view of the COVID-19 crisis.

In the recent years wake turbulence separations have been readjusted for new aircraft types ${ }^{3,4}$, new arrival procedures $^{5}$, and modified aircraft categories ${ }^{6,7}$. Whereas an unprecedented test program comprising many different phases of flight was accomplished for the determination of appropriate separation standards of the A380, other new developments focused mainly on wake vortex evolution in ground proximity ${ }^{8}$. The B747-8 lidar measurement program conducted wake measurements at one wing span above ground, because this altitude was considered the most hazardous scenario ${ }^{4}$. Lidar measurements employed for the development of the time-based separations procedures at London Heathrow airport also focused on the same flight altitude ${ }^{5}$. The time-based separations concept helps to maintain landing rates in strong headwinds by adjusting aircraft separations according to the current headwind strength.

The rationale for this substantial constraint of flight altitudes is that most encounters occur below $300 \mathrm{ft}$ above ground $^{9,10}$. In ground proximity vortex descent is restricted and the vortices tend to rebound due to the interaction with the ground surface ${ }^{11}$. Weak crosswinds may compensate the self-induced lateral propagation of the upwind vortex, such that it may hover over the runway directly in the flight path of the following aircraft ${ }^{12,13}$. As a consequence aircraft experience wake vortex encounters time after time, even under adherence to separation standards ${ }^{14}$. During final approach, pilots usually respond to encounters by initiating go-arounds, since their options to counteract the imposed rolling moment and re-stabilize the approach are restricted at low heights above ground.

The same flight height restriction has been applied and will apply to the aircraft wake turbulence recategorization initiative (RECAT) of ICAO. RECAT Phase 1 classifies aircraft types into six categories considering besides the weight the approach speed, wing characteristics and in parts also the rolling moment exerted on following aircraft. RECAT-1 has been implemented in November 2012 at Memphis airport ${ }^{6}$, followed by numerous other US airports. The new European separation standard for aircraft wake turbulence called RECAT-EU has been first introduced for arrivals and departures at Paris Charles de Gaulle airport in March 2016 followed by the airports London-Heathrow, Leipzig/Halle, and Toulouse ${ }^{7}$. The long-term goal of the RECAT initiative is to introduce dynamic pair-wise separations that consider the aircraft type pairing (pair-wise) and the environmental conditions that control wake vortex transport and decay (dynamic). In SESAR2020 the envisaged developments for static pairwise separations and weather dependent reduced wake turbulence separations for arrival and departure also focus on wake evolution in ground proximity.

To mitigate the risk of wake encounters during final approach and, in a next step, to improve runway capacity, so-called plate lines have been developed ${ }^{15}$. Each plate line consists of several upright plates that are installed beyond the ends of runways underneath the approach glide path perpendicular to the runway centerline. When wake vortices shed by landing aircraft approach the plate line, secondary vortices detach from the individual plates' surfaces, actively approach the primary vortices, and interact with those such that the wake vortices are weakened and their lifetime is reduced. Previous investigations of the plate line principle comprise towing tank experiments ${ }^{17}$, large eddy simulations ${ }^{16,17,18}$, and flight experiments with DLR's research aircraft $\mathrm{HALO}^{15}$. Encounter simulations employing LES flow fields reveal a significant reduction of the maximal vortex impact on the encountering aircraft after the installation of plate lines ${ }^{19}$.

Here we report the first installation of plate lines at an international airport and simultaneous measurements of the wake vortices with three lidars complemented by a comprehensive suite of meteorological instrumentation. Section II. provides a survey on previous investigations of plate line effects and introduces the underlying fluiddynamic principle. Section III. describes the acquired plate line design and how the compliance with airport requirements was assured in order to get approval of authorities for the installation of the plate lines at Vienna International Airport. In section IV. a survey on the positioning of the plates and the instrumentation at runway 16 is given. Section V. provides a detailed analysis of the measurement results starting with an outline of the developed evaluation strategy followed by descriptions of the effects of headwind strength, turbulence intensity, and aircraft types on the reduction of wake vortex lifetime and circulation strength. The section concludes with an analysis of the number of plate lines required to cover the area from where wake vortices may rebound into the flight corridor of aircraft during final approach. A preliminary analysis of the measurement data has been reported in Ref. 20 together with a more detailed description of the demonstration of frangibility of the elaborated plate design. 


\section{Previous Investigations of Plate Line Effects}

Before the current project, the plate line principle has been thoroughly investigated. First, fundamental research was conducted employing a towing tank through which a simplified aircraft model was towed and the flow was visualized with dye ${ }^{17}$. At that time, a rod-shaped massive obstacle was placed at the ground to trigger premature vortex decay. Quantitative measurements were conducted with particle image velocimetry indicating substantially accelerated vortex decay rates above and close to the obstacle at the ground ${ }^{17}$.

Large eddy simulations (LES) were used to better understand the underlying vortex dynamics ${ }^{17}$. When the wake vortices get close to the obstacle, strong secondary vortices are shed from the obstacle's surface that actively approach the wake vortices (see Figure 1, left). Then the secondary vortices are wrapped around the wake vortices by the primary wake vortex flow (Figure 1, center). Finally, the secondary vortices propagate actively along either side of the wake vortices and accelerate their decay (Figure 1, right).

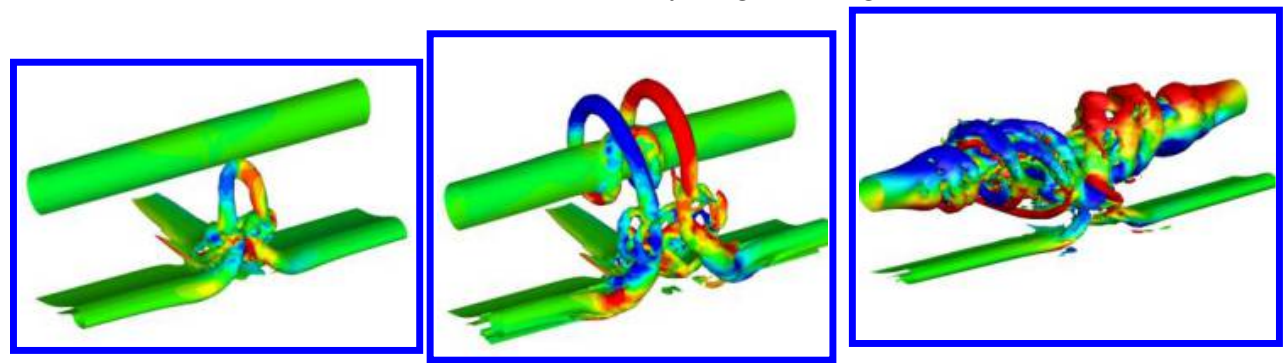

Figure $1 \Omega$-shaped secondary vortex approaches wake vortex and wraps around it leading to premature wake vortex decay.

In order to enable an installation at an airport, the massive obstacle was replaced by a series of plates, where the individual plates are aligned with the flight direction. LES showed that the effects of a plate line are even superior to those of a massive obstacle which can be attributed to the increased surface roughness of the plates compared to the smooth obstacle $^{16}$. LES were used to optimize the plate shape ${ }^{18,21,22}$ and to investigate the impact of crosswind and headwind ${ }^{16}$. As a result, a plate line with optimized plate dimensions of $4.5 \mathrm{~m}$ height and $9 \mathrm{~m}$ length, consisting of 8 plates and a plate separation of $20 \mathrm{~m}$ was designed. A respective patent has been filed under number DE 102011 010147 followed by international patents in the countries Austria, France, Great Britain, Japan, Singapore, and Switzerland. The patent was refused in the USA.

Finally, flight experiments were conducted with the DLR research aircraft HALO (Gulfstream G550) at special airport Oberpfaffenhofen ${ }^{15}$. HALO was flying traffic patterns in high-lift configuration at a constant height of about one initial vortex separation $b_{0}=22 \mathrm{~m}$ over a single plate line. The plate line consisted of six wooden plates that were erected laterally displaced from the runway. Lidar measurement results indicated that the lifetime of the longest-lived and thus potentially most hazardous wake vortex could be reduced by one third ${ }^{15}$.

\section{Plate Design and Approval of Authorities}

Several potential plate designs have been developed prior to the installation at Vienna airport. For example, a permanent design consisting of aluminum lattice masts certified for airports covered by truck tarpaulin and fixed to the ground by concrete foundations. Another low-cost, robust, and safe alternative were thuja hedges where the wind force would be distributed on many flexible stems. However, Vienna airport opted for the development of a temporary plate line design that can be removed easily after the live trial. A permanent design should only be considered after the benefits of the plate lines have been demonstrated. 

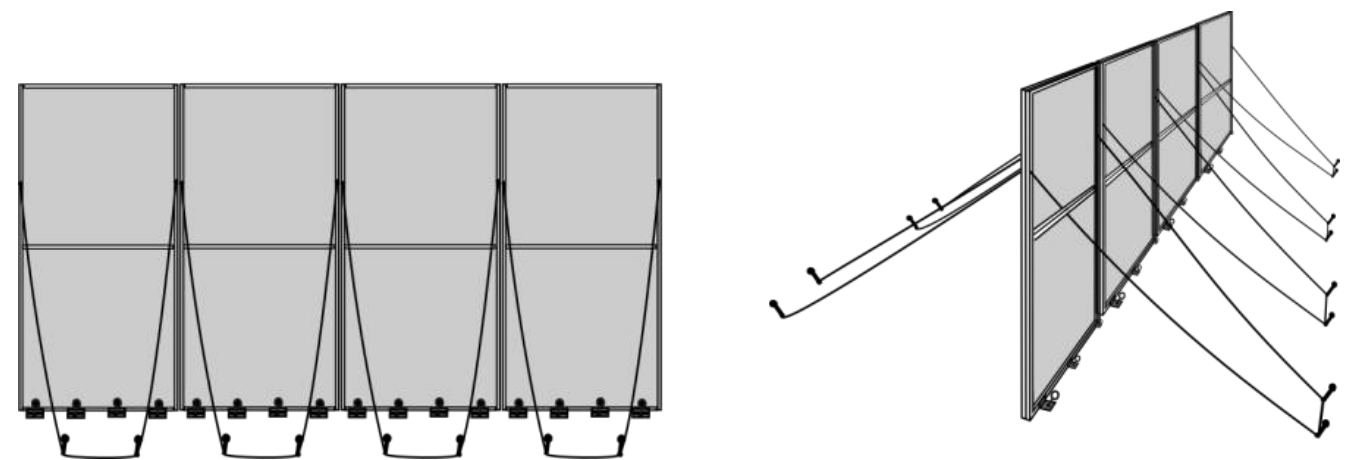

Figure 2 Plate line design for temporary installation.

The developed plate design for temporary installation consists of four wooden frames covered by truck tarpaulin with a height of $4.5 \mathrm{~m}$ and a length of $2.25 \mathrm{~m}$, respectively (see Figure 2). A complete plate consisting of four elements has the dimensions of $4.5 \mathrm{~m}$ height and $9 \mathrm{~m}$ length. The plates are manufactured with hinges such that they can be folded up and down and secured at the ground when they are not used. The plates and the synthetic ropes are fixed to the ground with ground anchors.

The plates were designed for a maximum wind speed of $20 \mathrm{~m} / \mathrm{s}$ with a safety factor of $\mathrm{S}=3$. A stability test with a plate prototype during cyclone Friederike on 17 to 18 January 2018 showed that the plate resists winds of $15 \mathrm{~m} / \mathrm{s}$ $(54 \mathrm{~km} / \mathrm{h}, \mathrm{Bft} 7)$ measured at $2 \mathrm{~m}$ above ground. An independent expert assessment confirmed the stability analysis regarding wind forces, the stability analysis of the ground anchors, and conducted a geotechnical assessment of the soil at runway 16 of Vienna airport. Finally, an assessment of the secured plates for winds of $200 \mathrm{~km} / \mathrm{h}$ was conducted in a wind tunnel to make sure that the secured plates would also resist heavy storms.

The University of Braunschweig proved the electromagnetic compatibility with the ILS localizer conducting measurements at scale 1:144. They found that even disturbances by steel plates are well within ICAO CAT III tolerances $^{23}$. Nevertheless, it was decided to install the first plate line behind the localizer in order to avoid any impairment of the ILS signal which appeared to be already close to the tolerances due to other disturbances.

In order to comply with the frangibility requirements the plates feature predetermined breaking points in terms of dimensions of screws, metal brackets and anisotropic cross-sections of the wooden frames. Frangibility was demonstrated with a plate prototype according to the Autodrome Design Manual, Part 6 Frangibility of ICAO ${ }^{24}$. For this purpose a steel tube with outer diameter of $250 \mathrm{~mm}$, mimicking the leading edge of a wing, was mounted in $4 \mathrm{~m}$ height at the plate prototype. The steel tube was connected by a rope with a van that accelerated to about $35 \mathrm{~km} / \mathrm{h}$ before the rope got strained (see Figure 3 in Ref. 20). A weak link with a breaking force of $10 \mathrm{kN}$ connected with the rope made sure that the prescribed maximum force of $45 \mathrm{kN}$ was not exceeded when the abruptly accelerated steel tube broke the plate prototype.

The plate lines were classified as air traffic control system. They comply with the requirements set forth by ICAO regarding obstacle clearance, stability, and frangibility. Substantial documentation of the plate design and placement, of the involved instrumentation and the procedures during the measurement campaign was produced. Based on this a safety assessment was conducted by the EASA Safety- \& Compliance Management of Vienna Int. Airport assuring that the plate lines comply with the relevant standards and regulations. Finally, the installation of the plates and the instrumentation was approved by the Austrian authorities (Bundesministerium für Verkehr, Innovation und Technologie, BMVIT). An Aeronautical Information Circular was established to inform the pilots without receiving any feedback. The measurement campaign started on 6 May 2019 and was completed on 28 November 2019.

\section{Positioning of Plate Lines and Instrumentation}

Figure 3 shows the positioning of the plate lines (red dashes) and the instrumentation installed for the measurement campaign. The first plate line, consisting of 8 plates separated by $20 \mathrm{~m}$, is situated at a distance of about $400 \mathrm{~m}$ to the threshold of runway 16 . The two innermost plates are rotated by $13^{\circ}$ to avoid that the visibility of the approach lighting could be obscured. The second plate line consisting of 9 plates is placed at a distance of $320 \mathrm{~m}$ to plate line 1. Nine plates were chosen to avoid placing one of the plates on the road way. 


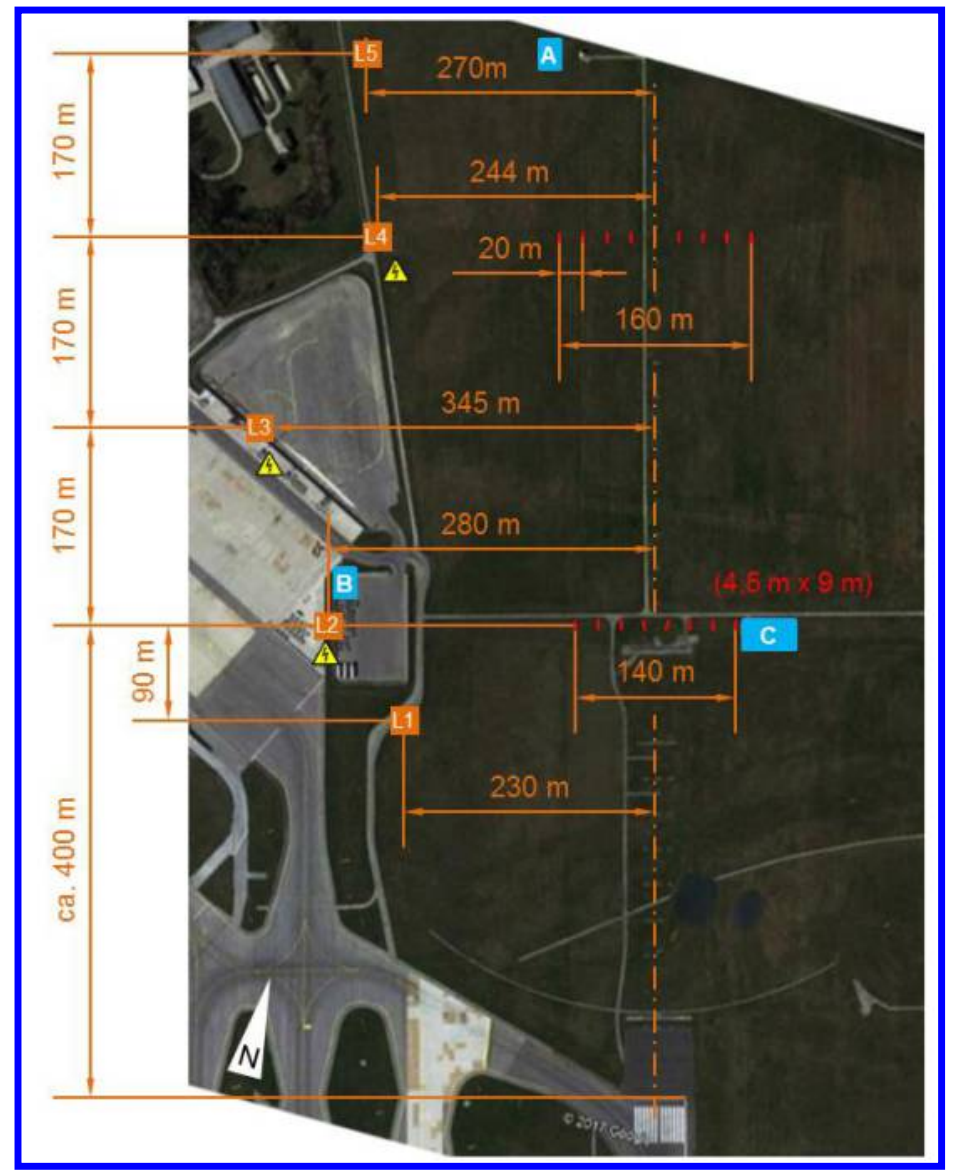

Figure 3 Positioning of plate lines and instrumentation (@) Google 2017).

Figure 3 shows the five measurement positions L1 - L5 of the lidars of type Leosphere Windcube 200S. The lidars were scanning in vertical planes perpendicular to the flight direction ${ }^{26}$. Average flight altitudes above ground at the lidar planes L1 - L5 were determined from Mode-S data to $40.8 \mathrm{~m}, 45.8 \mathrm{~m}, 54.3 \mathrm{~m}, 64.8 \mathrm{~m}$, and $74.5 \mathrm{~m}$, respectively, with a standard deviation of $4.9 \mathrm{~m}$.

Lidar measurement planes L2 and L4 characterize wake vortex decay directly above a plate line for two different flight altitudes. Measurement plane L1 captures the interaction of the disturbances stemming, on the one hand, from touchdown (so-called end effects ${ }^{15,25}$ ) and, on the other hand, from the plates. Plane L3 measures the interaction of the disturbances spreading from the two plate lines, whereas L5 investigates the propagation of plate line disturbances against flight direction. Because only three lidars were available for the five measurement planes, three combinations of measurement planes were operated subsequently: (L1, L2, L3), (L2, L3, L4) and (L3, L4, L5). Measurements were conducted alternatingly with erected plates on one day and without plates on other days.

For a comprehensive wake vortex decay assessment, weather conditions have to be taken into account, which have a major impact on the lifetime of the vortices. Therefore, the following additional meteorological sensors were installed for this campaign. Positions A and B in Figure 3 were equipped with the transmitters and receivers of the RPG (Radiometer Physics GmbH) microwave scintillometer and the Kipp \& Zonen optical scintillometer. The combination of these instruments enables synchronous measurements of latent and sensible heat fluxes in the atmospheric surface layer. From the scintillometer data also the structure parameter $C_{n}^{2}$ can be computed which is used here to derive different turbulence regimes. Position B hosted the RPG Doppler Cloud Radar RF Band determining speed of cloud particles, raindrops, snow, and insects. At the shelter in position C, the RPG HATPRO Microwave Radiometer (vertical profiles of temperature and humidity, liquid water path), the Axis M3027-PVE Fisheye Camera (optical cloud observation) and the FLIR Infrared Camera (infrared radiation) were installed. Further, on the roof top of parking garage 4 the Leosphere Windcube 400S-AT wind lidar (wind speed) and the LEONARDO Rainscanner 3D weather radar were placed (wind speed, hydrometeor classification). 


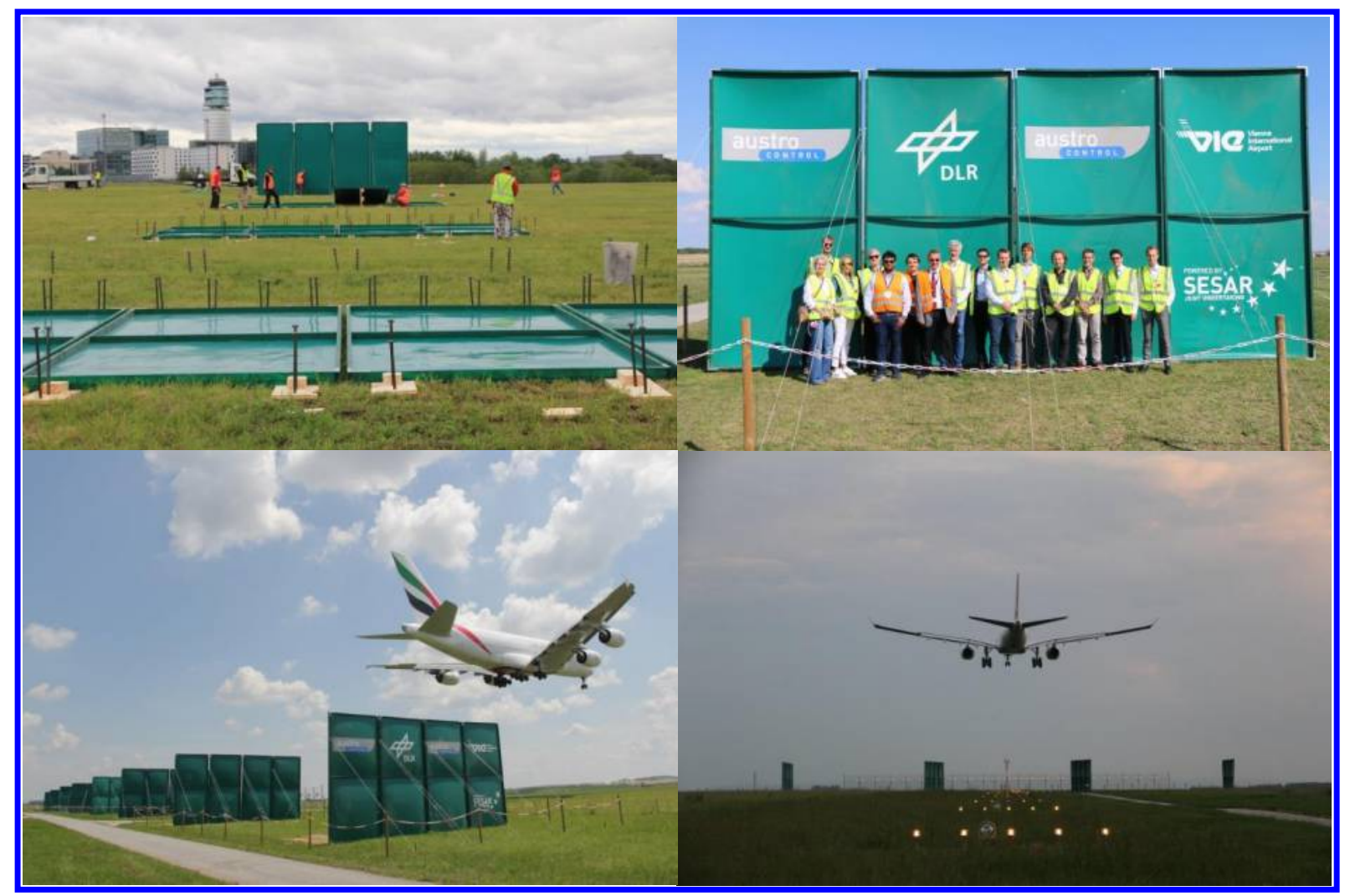

Figure 4 Impressions of the installation of plate line 2, the visitors' day, and overflights of plate line 1 (in clockwise order).

The plates were manufactured in green color such that they could not be easily discriminated from the surrounding grass land. A pilot reported that the erected plates were barely visible from the cockpit of an aircraft on approach and he was arguing whether the plates were already removed when he tried to spot them. Figure 4 shows impressions of the installation of plate line 2, the visitors' day, and overflights of plate line 1 located at the localizer (in clockwise order).

\section{Life Trial Results}

The measurement data used for the current analysis was collected within a time frame ranging from 6 May 2019 to 28 November 2019. On 21 days measurements with plate lines took place and 45 days saw measurements without plates. During that time frame 9,473 approaches on runway 16 were measured. From these 235 approaches with plates were processed corresponding to 589 individual vortex evolutions. Without plates 266 approaches were processed with 637 individual vortex evolutions.

\section{A. Evaluation Strategy}

An evaluation strategy has been developed focusing on safety aspects. For this purpose the data is assessed and filtered in various ways.

Quality Control: The lidar data is processed interactively employing an algorithm further developed in-house based on the method described in Ref. 26. During manual processing, the software doesn't indicate for a particular case whether the plates were folded up or down. The resulting vortex properties (lateral position, vertical position, and circulation against time) are subsequently quality controlled by visual inspection and erroneous data points are excluded. Such erroneous data points may occur at a progressed state of vortex decay where the coherent structure of the vortices is getting lost and the vortices cannot be differentiated easily from atmospheric eddies.

Safety Corridor: Only wake vortices residing within a safety area of $\pm 50 \mathrm{~m}$ from the runway centerline are considered relevant, whereas vortices permanently transported out of that safety area are neglected for the analysis. 
The dimensions of the safety corridor are inspired by the definition of a safety area of a runway of the Frankfurt wake vortices warning system WVWS ${ }^{27}$. The WVWS assumes a maximum lateral deviation of the aircraft from the extended centerline of $\pm 15 \mathrm{~m}$ plus a critical gap of $\pm 30 \mathrm{~m}$ between the vortex core and the aircraft fuselage. The resulting safety corridor of $\pm 45 \mathrm{~m}$ is rounded up to $\pm 50 \mathrm{~m}$.

Wind Effects: At the airport London Heathrow most encounters have been observed for crosswinds below 2 knots and headwinds next to zero 9 . With increasing headwinds so-called end effects are transported ever faster towards the lidar measurement planes accelerating vortex decay ${ }^{15}$. As a consequence, various headwind ranges have been considered in the data analysis but the main analysis focuses on headwinds lower than $2 \mathrm{~m} / \mathrm{s}$. This includes also cases with tailwinds where an accumulation of encounters was observed at Vienna airport ${ }^{14}$. With increasing crosswinds the vortices are quickly advected out from the safety corridor such that no crosswind filtering is needed. The wind data consists of $2 \mathrm{~min}$ averages taken from the anemometer of runway 16 (WMA16) situated about $760 \mathrm{~m}$ from plate line 1.

Aircraft Type Effects: To enable the comparison of wake vortices generated by different aircraft types, the data characterizing vortex decay is partly normalized (made dimensionless). Normalized quantities are denoted by an asterisk (*). The vortex circulation (strength) data is normalized by its initial value $\Gamma_{0}=\mathrm{mg} / \rho \mathrm{b}_{0}$ TAS. The required aircraft types and values of true airspeed (TAS) in the lidar measurement planes are taken from the Mode-S protocol. Air density $\rho$ is retrieved from pressure and temperature also from the Mode-S protocol. The initial vortex separation is computed according to $\mathrm{b}_{0}=\pi / 4 \mathrm{~B}$ assuming elliptical circulation distribution, where $\mathrm{B}$ is the wing span gathered from the BADA (Base of Aircraft Data) data base ${ }^{28}$. Austrian Airlines kindly provided the landing weight, $\mathrm{m}$, of their fleet for all recorded landings with lidar measurements. In all remaining cases the landing weight was estimated according to $m=\left(\frac{T A S \sqrt{\rho / \rho_{0}}-V d_{D E S}}{C_{V \min } \cdot V_{\text {stall,ref }}}\right)^{2} m_{\text {ref }}$ following BADA ${ }^{28}$, where $\rho_{0}$ is the density of air at sea level of the International Standard Atmosphere, the descent speed increment $\mathrm{Vd}_{\mathrm{DES}}$ amounts to 5 knots below $1000 \mathrm{ft}$, $\mathrm{C}_{\mathrm{V} \min }=1.3, \mathrm{~V}_{\text {stall,ref }}$, is the stall speed for the landing configuration of the particular aircraft type and $\mathrm{m}_{\text {ref }}$ its respective reference mass. To avoid unrealistically high landing masses the estimated mass was clipped off at $95 \%$ of the maximum landing weight. The characteristic vortex time scale $t_{0}$ is used to normalize vortex age. $t_{0}$ corresponds to the time the wake vortices initially need to descend one vortex separation $b_{0}$ and is calculated according to $\mathrm{t}_{0}=2 \pi \mathrm{b}_{0}^{2} / \Gamma_{0}$.

Circulation Reduction: In order to quantify the degree of circulation decay with and without the plate lines $25^{\text {th }}$, $50^{\text {th }}$ (median), $75^{\text {th }}, 95^{\text {th }}$, and $100^{\text {th }}$ (maximum) percentiles are employed. Vortex-age-binned percentiles are computed with dimensional bin widths of $20 \mathrm{~s}$. For example, this provides circulation values for the five selected percentiles in a vortex age frame of $50 \mathrm{~s} \pm 10 \mathrm{~s}$ (see green rectangles in Figure 5, center-right and Figure 6, topright). For this analysis vortices, where the circulation can no longer be determined due to a progressed state of vortex decay, are assigned a circulation value of zero. This avoids that vortices are falling out of the samples and thus allows assessing unbiased circulation decay statistics. Circulation reduction can only be evaluated if the vortices are considered throughout their entire lifetime. The latter criterion is met by considering only vortices where the last measured location is situated within the safety corridor.

Lifetime Reduction: Additionally, circulation-binned percentiles are used to extract different percentiles of vortex lifetimes for given circulation strengths (see blue rectangle in Figure 5, center-right and Figure 6, bottom- right). The circulation bin widths are adjusted to $50 \mathrm{~m}^{2} / \mathrm{s}\left( \pm 25 \mathrm{~m}^{2} / \mathrm{s}\right)$. Normalized bin widths are adjusted to one vortex time scale $\mathrm{t}_{0}\left( \pm 0.5 \mathrm{t}_{0}\right.$, orange rectangle).

Statistical Hypothesis Testing: The Wilcoxon rank-sum test is a non-parametric statistical hypothesis test used to compare the two circulation-binned probability density distributions (PDDs) for the dimensional vortex age in order to assess whether or not they differ in statistically significant ways ${ }^{29}$. The null hypothesis of the Wilcoxon rank-sum test is that the PDDs from cases with and without plate lines are equal, i.e. the plate lines have no effect on the vortex age distributions. The alternative hypothesis is that the PDDs with and without plate lines are shifted in location, i.e. the observed vortex ages for cases with plate lines deviate from the cases without plate lines. The Wilcoxon rank-sum test yields a p-value representing the probability of obtaining the observed results under the null hypothesis. A p-value below the selected significance level of $\alpha=0.05$ indicates that the null hypothesis should be rejected and that the effects of the plate lines are statistically significant. Further, 95\%-confidence intervals for the change in median vortex lifetime effected by the plate lines are estimated using the Wilcoxon rank-sum test. The Wilcoxon test is conducted employing the R programming language for statistical computing ${ }^{30}$. 


\section{B. Analysis of Wake Vortex Behavior}

Figure 5 depicts the wake vortex behavior of 472 landings consisting of a mix of 13 different aircraft types of the category Heavy (H) plus one Super (J, A380) and two Medium (M) aircraft types. One landing was measured in at least one and up to three of the considered lidar planes L1 to L5 (see Figure 3). Combining the data of the different lidar planes follows the rationale that wake vortex behavior shall be made safer anywhere where wake vortices might rebound to the flight path and that the statistics become more significant the larger the data sample.

In Figure 5 measurements with plate lines (PL) are denoted by dots in cold colors: green dots for the port vortex and blue dots for the starboard vortex. Warm colors are used for the reference cases without plates: red dots for the port vortex and magenta dots for the starboard vortex. The individual panels show vortex height $\mathrm{z}$ against vortex age $\mathrm{t}$ (top left), vortex height $\mathrm{z}$ over vortex lateral position $\mathrm{y}$ (top right, $\mathrm{y}=0$ corresponds to the extended runway centerline), vortex lateral position y against vortex age $\mathrm{t}$ (center-left), vortex circulation $\Gamma$ over vortex age $\mathrm{t}$ (centerright), lateral wind speed $\mathrm{v}$ (positive away from lidar) over longitudinal wind speed $\mathrm{u}$ (positive in flight direction) measured by anemometer WMA16 and derived from Mode-S data in the five lidar measurement planes (bottom left), and normalized vortex circulation $\Gamma^{*}$ over normalized vortex age $t^{*}$ (bottom right).
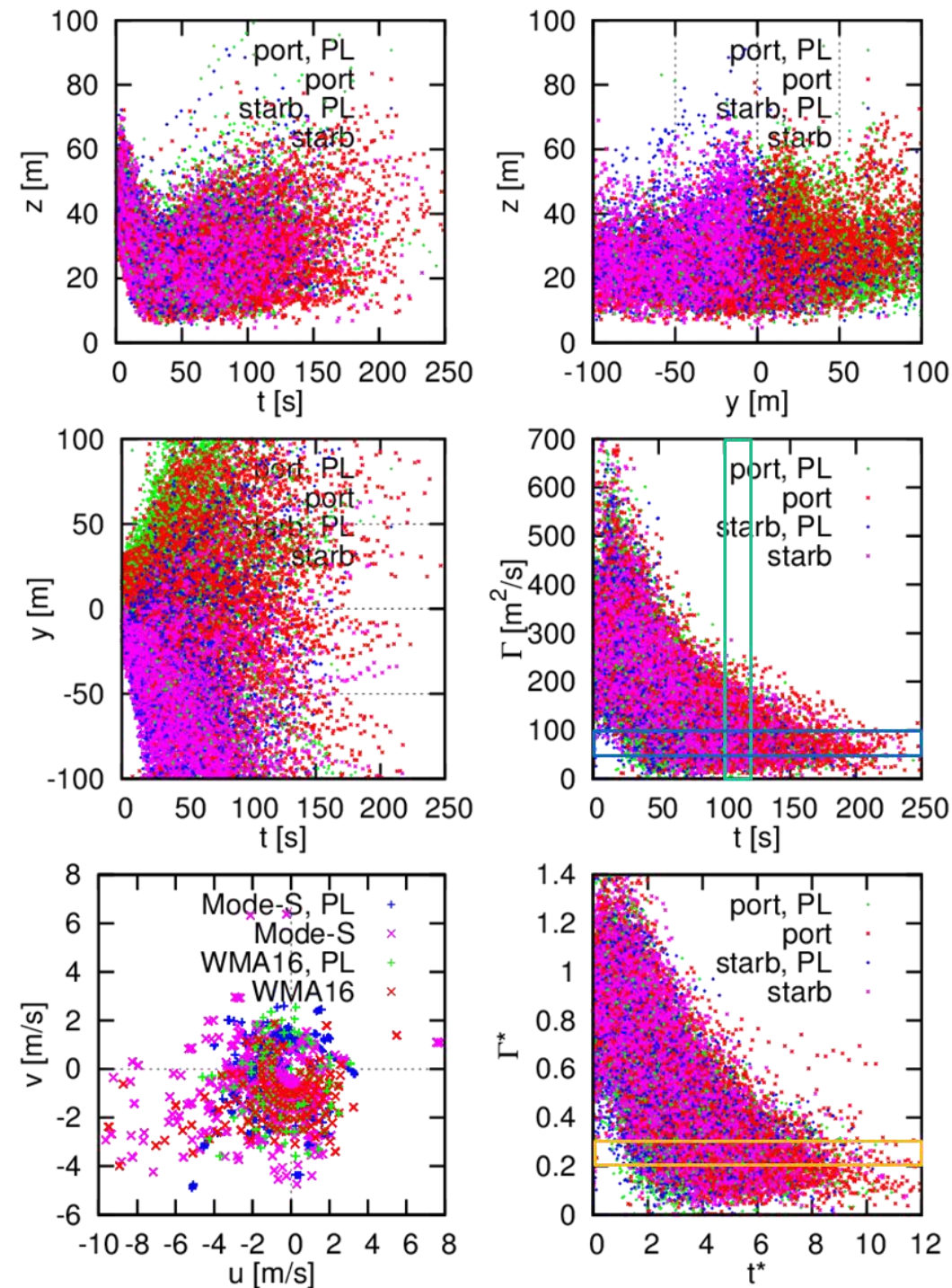

Figure 5 Wake vortex measurements of 206 landings / 503 vortex evolutions with and 266 landings / 548 vortex evolutions without plate lines (weight classes J, H, M). Bottom left prevailing wind conditions. 

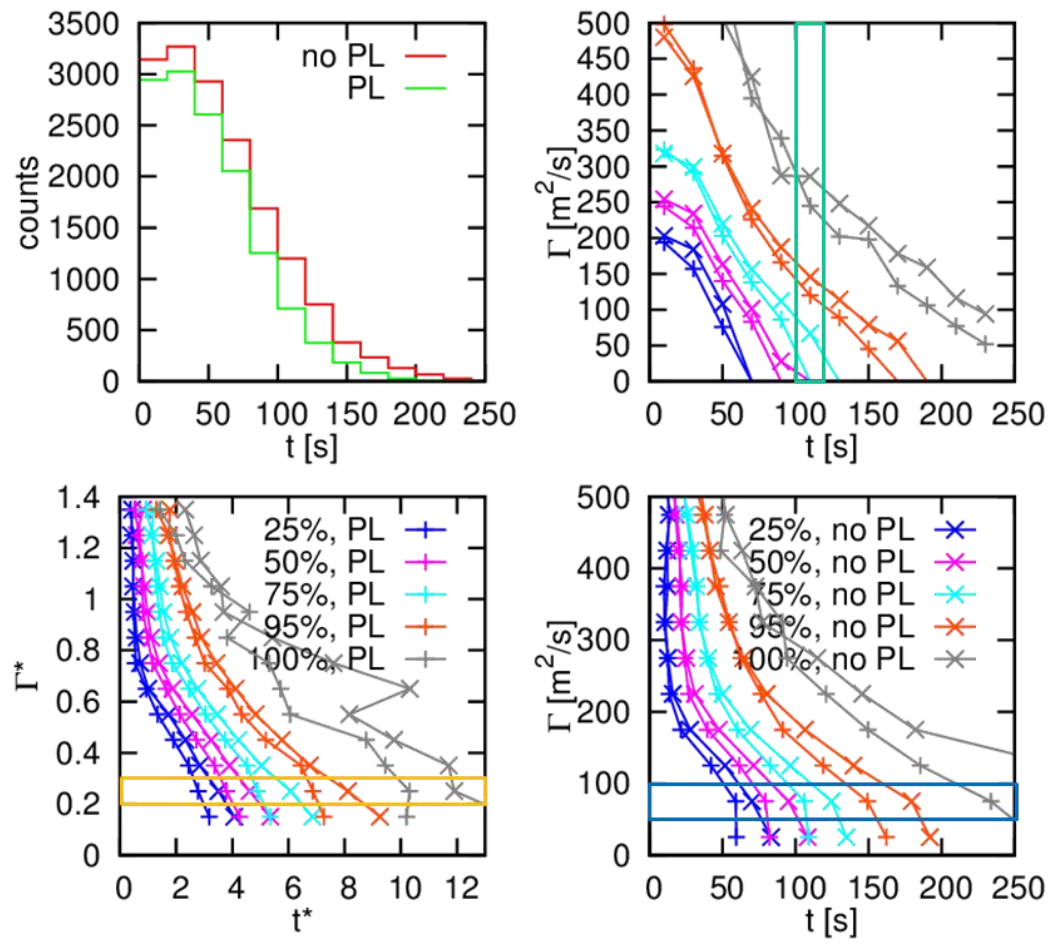

Figure 6 Vortex measurement counts (top left), vortex-age-binned circulation percentiles (top right), and circulation-binned normalized (bottom left) and dimensional (bottom right) vortex age percentiles of 472 landings (weight classes J, H, M).

The scatter plots don't allow seeing the plate line effects clearly. Figure 6 shows various percentiles characterizing vortex decay of the 472 landings displayed in Figure 5. The counts of vortex measurements with and without plates in 20 s-bins in Figure 6 top left make the vortex mitigation effects intuitively accessible. For quantification of the plate line effects we employ the time-binned circulation percentiles (Figure 6 top right) as well as the circulation-binned vortex age percentiles (Figure 6 bottom) as described under the keywords "Circulation reduction" and "Lifetime reduction" in section V.A, respectively.

The vortex-age-binned circulation percentiles (top right) rate the circulation reduction for different vortex age bins. Two vortex age bins between $100 \mathrm{~s}$ and $140 \mathrm{~s}$ were selected in order to represent the effects within the range of the most relevant ICAO separation Medium behind Heavy of $5 \mathrm{NM}$ corresponding to about $120 \mathrm{~s}$. For the vortex age bin ranging from 100 to $120 \mathrm{~s}$ denoted by the green rectangle, the medians of both magenta colored curves already amount to zero. This means that in more than $50 \%$ of the cases circulation can no longer be determined, i.e. the vortices can be considered to be fully decayed. For the $75^{\text {th }}$ percentile without plate lines $\Gamma$ amounts to $67 \mathrm{~m}^{2} / \mathrm{s}$, while it amounts to zero with the plates. That is, with plates more than $75 \%$ of the cases have fully decayed. This corresponds to a circulation reduction of $100 \%$ for the $75^{\text {th }}$ percentile. For the $95^{\text {th }}$ percentile the circulation drops from $146 \mathrm{~m}^{2} / \mathrm{s}$ without plates to $120 \mathrm{~m}^{2} / \mathrm{s}$ with the plates leading to a circulation reduction of $18 \%$.

The percentiles in Figure 6 bottom reveal the reduction of vortex lifetimes within circulation bins. At high circulations values the curves with and without plates fall on top of each other. When the plate effect starts to accelerate vortex decay, the curves start to deviate. So the circulation-binned curves feature a $\lambda$-shape.

The circulation-binned approach allows for a quantitative analysis of the vortex lifetime reduction. As an example, consider the dimensional circulation-binned vortex age values for the $95^{\text {th }}$ percentile, indicated in orange in the bottom-right chart in Figure 6. For vortices in the circulation range between 50 and $100 \mathrm{~m}^{2} / \mathrm{s}$ denoted by the blue rectangle, the vortex age at the $95^{\text {th }}$ percentile without plate lines is $180 \mathrm{~s}$ (indicated by "x"). This means that $95 \%$ of the vortices observed at this circulation range have a vortex age of $180 \mathrm{~s}$ or fewer. With erected plate lines (indicated by "+"), the vortex age at the $95^{\text {th }}$ percentile is reduced to $149 \mathrm{~s}$. This corresponds to a vortex lifetime reduction of $17 \%$. 
Table 1 Reductions of wake vortex circulations and lifetimes of 472 landings (weight classes J, H, M).

\begin{tabular}{|c|c|c|c|c|c|}
\hline & Bins & $50^{\text {th }}$ percentile & $75^{\text {th }}$ percentile & $95^{\text {th }}$ percentile & average \\
\hline \multirow{2}{*}{$\begin{array}{c}\text { Dimensional } \\
\text { circulation reduction }\end{array}$} & $100-120 s$ & - & $100 \%$ & $18 \%$ & \multirow{2}{*}{$47 \%$} \\
\hline & $120-140 \mathrm{~s}$ & - & - & $22 \%$ & \\
\hline \multirow{2}{*}{$\begin{array}{l}\text { Dimensional } \\
\text { lifetime reduction }\end{array}$} & $0-50 \mathrm{~m}^{2} / \mathrm{s}$ & $24 \%$ & $19 \%$ & $16 \%$ & \multirow{4}{*}{$19 \%$} \\
\hline & $50-100 \mathrm{~m}^{2} / \mathrm{s}$ & $17 \%$ & $15 \%$ & $17 \%$ & \\
\hline \multirow{2}{*}{$\begin{array}{l}\text { Normalized lifetime } \\
\text { reduction }\end{array}$} & $0.1-0.2$ & $21 \%$ & $22 \%$ & $22 \%$ & \\
\hline & $0.2-0.3$ & $18 \%$ & $19 \%$ & $15 \%$ & \\
\hline
\end{tabular}

The circulation and lifetime reductions achieved at different percentiles are given in Table 1. Because only the stronger and longer-lived vortices are of interest, only values of the $50^{\text {th }}, 75^{\text {th }}$, and $95^{\text {th }}$ percentiles are considered. The $100^{\text {th }}$ percentile is omitted because it corresponds to a single measurement with little statistical significance. All values in Table 1 are positive which means that the plates reduce circulation and vortex lifetime. The achieved reductions range between $15 \%$ and $100 \%$. In order to characterize the reduction potential in a clear way the last column lists the average circulation reduction of here $47 \%$ and the average lifetime reduction of $19 \%$.
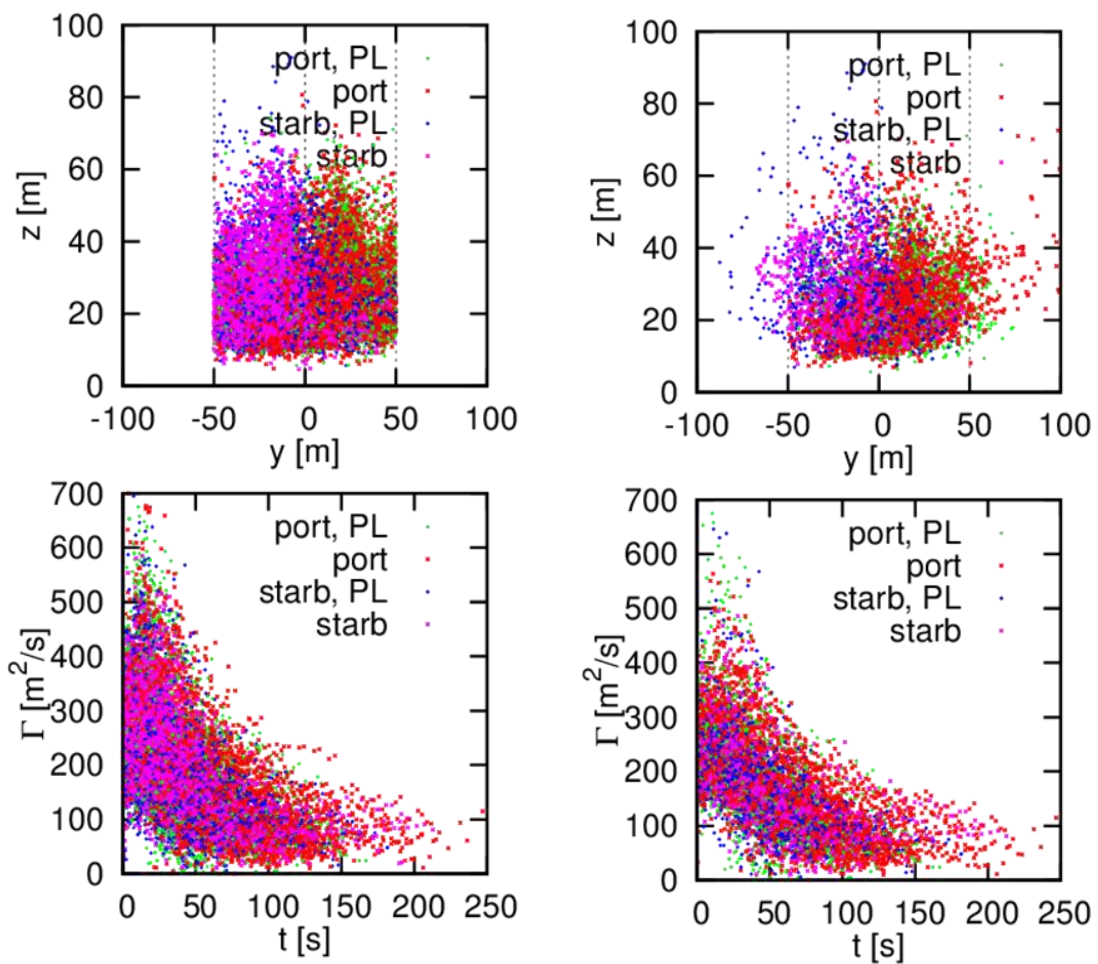

Figure 7 Wake vortex measurements (weight classes J, H, M) for headwinds lower than $2 \mathrm{~m} / \mathrm{s}$. Wake vortices residing within the $\pm 50 \mathrm{~m}$ safety corridor (left) and complete vortex evolutions where the last vortex observation lies within the safety corridor (right).

Following the arguments described under the keyword "headwind effects" in section V.A., Figure 7 displays approaches with headwinds below $2 \mathrm{~m} / \mathrm{s}$ as measured by the WMA16. These include cases with tailwinds up to $3 \mathrm{~m} / \mathrm{s}$. For this data sample Mode-S wind measurements nevertheless reach up to headwinds of $6 \mathrm{~m} / \mathrm{s}$. This indicates substantial variability in the wind field but also uncertainties of measurement accuracies in particular of the Mode-S wind data.

Figure 7 left considers only vortices residing in the safety corridor extending $\mathrm{y}= \pm 50 \mathrm{~m}$ from the runway centerline, whereas vortices transported out of that safety area are neglected. The plot contains data of 189 landings / 456 vortex evolutions with and 231 landings / 472 vortex evolutions without plate lines. The corresponding 
lifetime reductions by the installation of plate lines within the safety corridor are displayed in Figure 8 bottom and Table 2. Figure 7 left indicates that many vortices are quickly transported out of the safety corridor but that also a substantial part of the vortices remain in the corridor until final decay. The circulation decay curve indicates higher circulation values and longer lifetimes of the vortices developing over flat ground.

Figure 7 right contains instead vortex evolutions where the last vortex observation resides within the safety corridor $\left(\left|y_{\text {last }}\right|<50 \mathrm{~m}\right)$. While a number of vortices leave the safety corridor in between and then drift back into the corridor, this criterion makes sure that all vortices are considered throughout their entire observed lifetime. This criterion has to be met to estimate the circulation reduction potential of the plate lines. Figure 7 right is based on 243 vortex evolutions with and 226 vortex evolutions without plate lines.

The criteria $|\mathrm{y}|<50 \mathrm{~m}$ and $\left|\mathrm{y}_{\text {last }}\right|<50 \mathrm{~m}$ are satisfied by differing numbers of wake vortices. In order not to exclude meaningful parts of the measurements, we refrain from balancing exactly the numbers of the cases with and without plate lines. With as big as possible data samples the statistical significance of the analyses is strengthened.
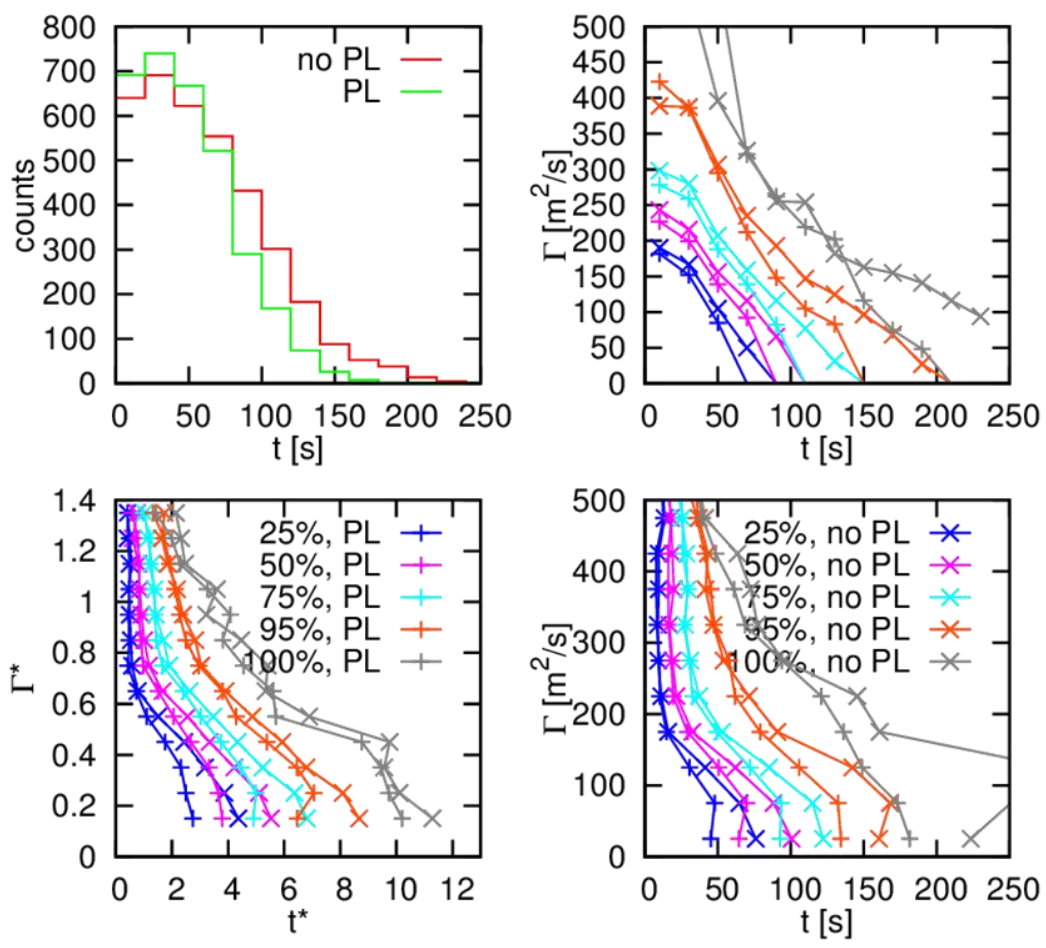

Figure 8 Vortex measurement counts (top left), vortex-age-binned circulation percentiles (top right), and circulation-binned normalized (bottom left) and dimensional (bottom right) vortex age percentiles (weight classes J, $\mathrm{H}, \mathrm{M}$ ) employing the $\pm 50 \mathrm{~m}$ safety corridor for headwinds lower than $2 \mathrm{~m} / \mathrm{s}$.

Applying the headwind filter and the $\pm 50 \mathrm{~m}$ safety corridor the percentile plots in Figure 8 reveal substantial circulation and lifetime reduction potential of the plate lines. The corresponding Table 2 features circulation reductions at vortex ages between $100 \mathrm{~s}$ and $140 \mathrm{~s}$ from $29 \%$ to $100 \%$ with an average of $66 \%$. Lifetime reductions of the long-lived vortices vary between $13 \%$ and $36 \%$ with an average of $24 \%$. The comparison of Table 1 and Table 2 indicates that focusing on weak headwinds and vortices within the safety corridor increases both the circulation and the lifetime reduction potentials.

Figure 9 depicts probability density distributions of the vortex lifetimes for different circulation ranges corresponding to the percentiles shown in Figure 8 bottom right. The PDDs for circulations above $250 \mathrm{~m}^{2} / \mathrm{s}$ with and without plate lines are almost identical indicating that the statistical basis for their further development is sound. While the p-value of the circulation bin $250 \mathrm{~m}^{2} / \mathrm{s}-300 \mathrm{~m}^{2} / \mathrm{s}$ still has a p-value of 0.9 and thus doesn't yet indicate any plate line effects on circulation decay, the p-values decrease substantially to 0.0003 for the $200 \mathrm{~m}^{2} / \mathrm{s}-250 \mathrm{~m}^{2} / \mathrm{s}$ bin. In Figure 8 bottom right this is exactly the circulation bin where the $\lambda$-legs start to deviate from each other. The lower circulation bins feature p-values between $10^{-21}$ and $10^{-30}$. This means that the PDDs with and without plate 
lines differ with extremely high statistical significances. Similar characteristics are also observed for the individual aircraft types described in section V.D.

The intervals in brackets listed in Table 2 denote the ranges of the $50^{\text {th }}$ percentiles (medians) estimated by the Wilcoxon rank-sum test with $95 \%$ confidence. The averages of these values are listed in the last column. These numbers estimate the uncertainties related to the lifetime reductions with plate lines and thus quantify, on one hand, the benefits that can be expected as a minimum and, on the other hand, the benefits that can be expected at best.

Employing a larger safety corridor of $\pm 60 \mathrm{~m}$ width reduces the circulation reduction from $66 \%$ to $50 \%$, whereas a smaller safety corridor of $\pm 40 \mathrm{~m}$ width only marginally increases the percentage to $67 \%$. These trends are expected, because previous numerical studies for the optimization of the plate line design indicated that additional plates ahead of a vortex strengthen the plate-induced vortex decay. On the other hand, the lifetime reductions don't seem to depend on these corridor width variations.

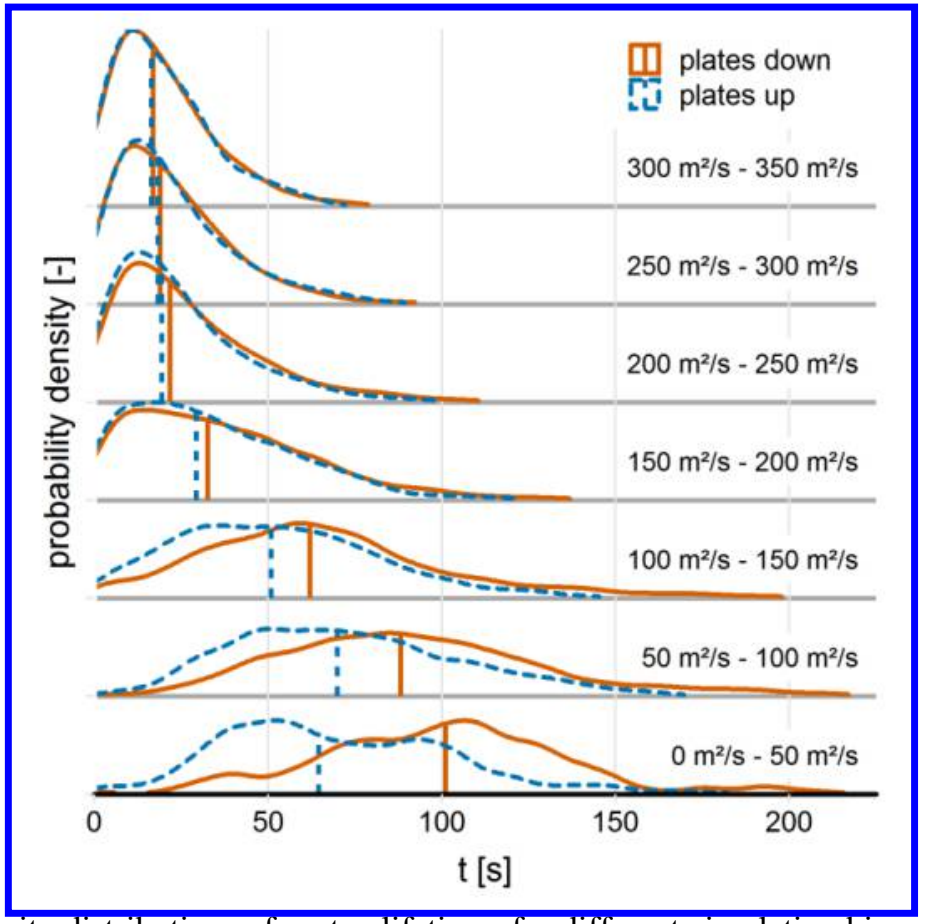

Figure 9 Probability density distributions of vortex lifetimes for different circulation bins corresponding to Figure 8 bottom right. Vertical lines indicate the median values.

Table 2 Reductions of wake vortex circulations and lifetimes (weight classes $\mathrm{J}, \mathrm{H}, \mathrm{M}$ ) with the $\pm 50 \mathrm{~m}$ safety corridor for headwinds lower than $2 \mathrm{~m} / \mathrm{s}$.

\begin{tabular}{cccccc}
\hline \hline & Bins & $50^{\text {th }}$ percentile & $75^{\text {th }}$ percentile & $95^{\text {th }}$ percentile & average \\
\hline Dimensional & $100-120 \mathrm{~s}$ & - & $100 \%$ & $29 \%$ & \multirow{2}{*}{$66 \%$} \\
circulation reduction & $120-140 \mathrm{~s}$ & - & $100 \%$ & $34 \%$ & \\
Dimensional & $0-50 \mathrm{~m}^{2} / \mathrm{s}$ & $36 \%(25 \%-36 \%)$ & $24 \%$ & $16 \%$ & \\
lifetime reduction & $50-100 \mathrm{~m}^{2} / \mathrm{s}$ & $21 \%(18 \%-25 \%)$ & $19 \%$ & $21 \%$ \\
Normalized & $0.1-0.2$ & $31 \%(27 \%-37 \%)$ & $28 \%$ & $26 \%$ \\
lifetime reduction & $0.2-0.3$ & $29 \%(24 \%-32 \%)$ & $22 \%$ & $13 \%$ & $24 \%$ \\
\hline
\end{tabular}

\section{Headwind Effects}

Figure 10 depicts dimensional and normalized circulation evolutions for different headwind ranges. The two stronger headwind ranges $\mathrm{u}>4 \mathrm{~m} / \mathrm{s}$ and $2 \mathrm{~m} / \mathrm{s}<\mathrm{u}<4 \mathrm{~m} / \mathrm{s}$ are almost exclusively populated by Heavy and Super 
aircraft. Therefore, the headwind effect is best comparable employing the normalized circulation evolutions displayed in the bottom panels of Figure 10. Despite much less data was processed for enhanced headwinds, it is obvious that vortex decay is well correlated with headwind speed. Maximum normalized vortex lifetimes are reduced from about 12 vortex time scales $t_{0}$ for weak headwinds to about $4 \mathrm{t}_{0}$ for headwinds above $4 \mathrm{~m} / \mathrm{s}$. With increasing headwind, disturbances caused by the touchdown of the aircraft, so-called end effects are transported ever faster into the measurement planes ${ }^{15}$. As a consequence, the current study focuses on weak headwinds below $2 \mathrm{~m} / \mathrm{s}$ where the most long-lived wake vortices pose the highest potential risks.

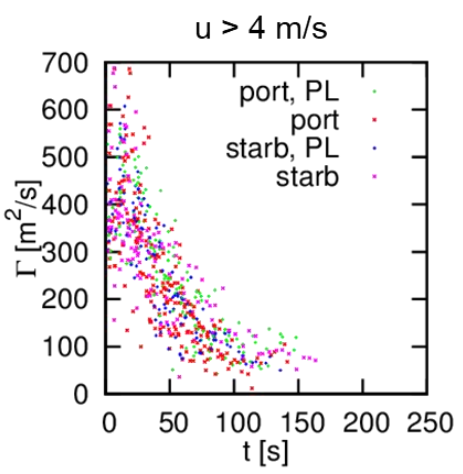

$2 \mathrm{~m} / \mathrm{s}<\mathrm{u}<4 \mathrm{~m} / \mathrm{s}$
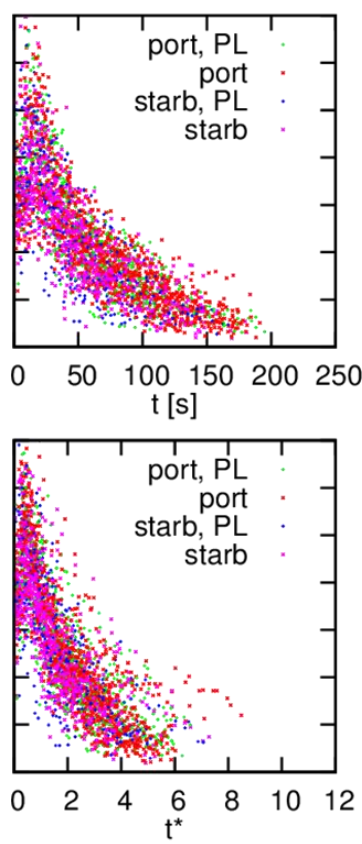

$0 \mathrm{~m} / \mathrm{s}<\mathrm{u}<2 \mathrm{~m} / \mathrm{s}$
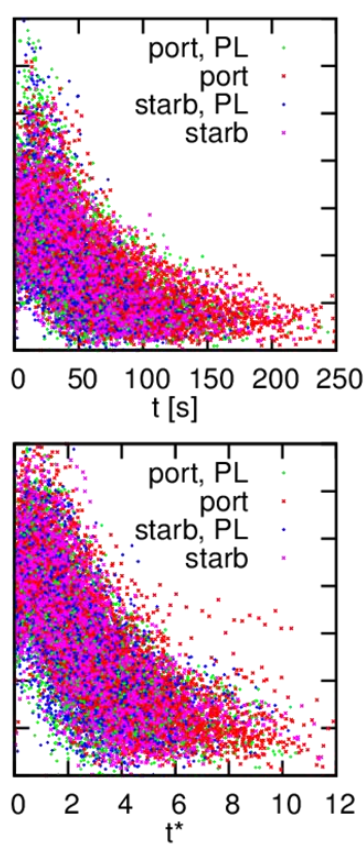

$-2 \mathrm{~m} / \mathrm{s}<\mathrm{u}<0 \mathrm{~m} / \mathrm{s}$
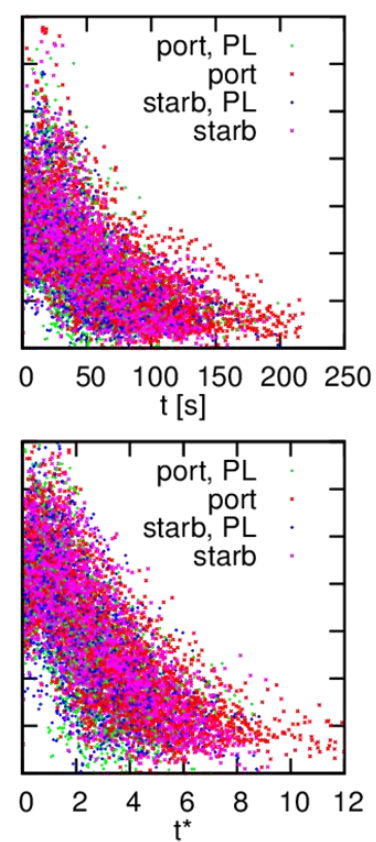

Figure 10 Dimensional (top) and normalized (bottom) circulation decay for various headwind ranges.

\section{Aircraft Type Effects}

The variability of vortex behavior can be substantially reduced by considering only wake vortices generated by a single aircraft type. With almost $28 \%$ of all landings the A320 constitutes by far the most frequent aircraft type at Vienna airport. As the A320neo (designated A20N) features the same maximum landing weight and wing span as the A320, it is added to the analysis of the A320 aircraft. As representative of the ICAO weight category Medium and category D "Upper Medium" of the RECAT-EU scheme, Figure 11 left depicts the wake vortex behavior of 348 landings of A320 and A20N aircraft in the $\pm 50 \mathrm{~m}$ safety corridor for headwinds lower than $2 \mathrm{~m} / \mathrm{s}$. In detail the data sample contains 145 approaches with plate lines corresponding to 337 vortex evolutions and 203 approaches without plates represented by 390 vortex evolutions. Remarkably, the longest-lived A320 vortices reach a maximum lifetime of over 200 s. Figure 11 right shows the subset of cases where the last measurement is situated within the safety corridor and is based on 200 vortex evolutions with and 200 vortex evolutions without plate lines.

For this smaller aircraft type circulation and lifetime reductions are less pronounced than for larger aircraft. Because Medium and Heavy aircraft do not require wake vortex separations behind A320 aircraft, the vortex agebinned circulation reduction for this Medium class aircraft (see Figure 12 top right) is considered around $60 \mathrm{~s}$ corresponding to radar separation. The average circulation reduction in Table 3 amounts to 8\%.

The lifetime reductions plotted in Figure 12 bottom clearly feature the $\lambda$-shape of the circulation-binned percentiles. Correspondingly, the PDDs of the lifetime reductions differ significantly for circulation values below $250 \mathrm{~m}^{2} / \mathrm{s}$ and $0.6 \Gamma^{*}$ according to the Wilcoxon rank-sum test. The circulation values, marking the onset of the plate line effects, appear the same for the other aircraft types considered below. Lifetime reductions in Table 3 vary between $4 \%$ and $40 \%$. The average of the lifetime reductions of the long-lived vortices (circulation bins $0-100 \mathrm{~m}^{2} / \mathrm{s}$ and $0.1-0.3$ ) amounts to $21 \%$, whereas the medians of the lifetime reductions with $95 \%$ confidence vary between $21 \%$ and $31 \%$. 

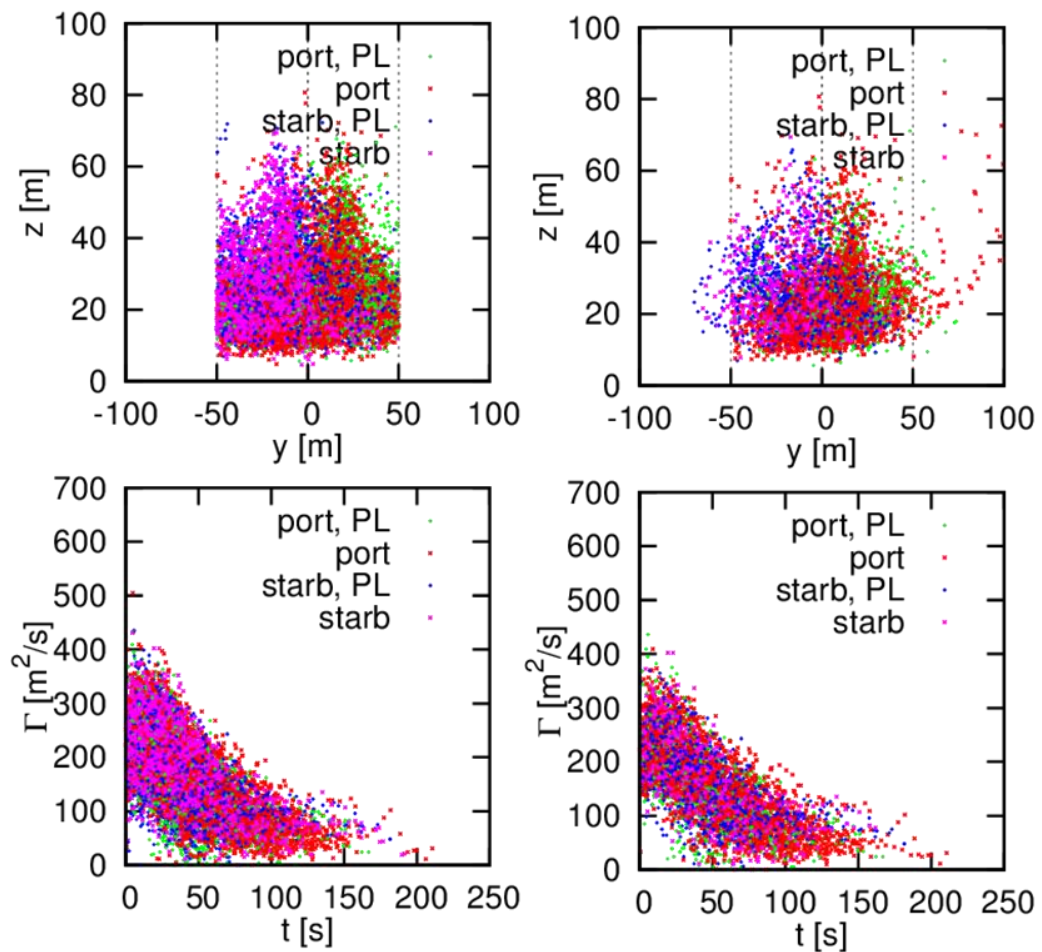

Figure 11 Measurements of A320 wake vortices for headwinds lower than $2 \mathrm{~m} / \mathrm{s}$. Vortices residing within the $\pm 50 \mathrm{~m}$ safety corridor (left) and complete vortex evolutions where the last vortex observation lies within the safety corridor (right).
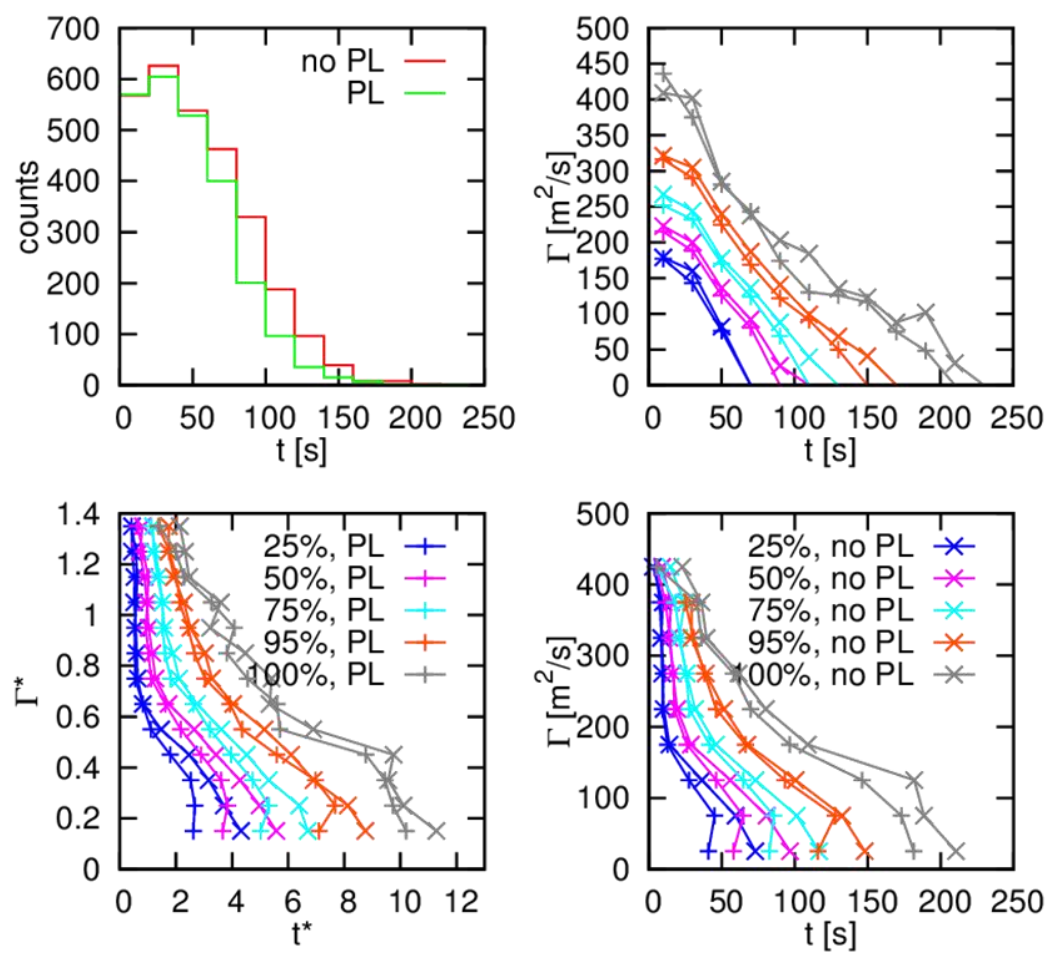

Figure 12 A320 vortex measurement counts (top left), vortex-age-binned circulation percentiles (top right), and circulation-binned normalized (bottom left) and dimensional (bottom right) vortex age percentiles. 
Table 3 Reductions of A320 wake vortex circulations and lifetimes with the $\pm 50 \mathrm{~m}$ safety corridor for headwinds lower than $2 \mathrm{~m} / \mathrm{s}$.

\begin{tabular}{|c|c|c|c|c|c|}
\hline & Bins & $50^{\text {th }}$ percentile & $75^{\text {th }}$ percentile & $95^{\text {th }}$ percentile & average \\
\hline \multirow{2}{*}{$\begin{array}{c}\text { Dimensional } \\
\text { circulation reduction }\end{array}$} & $40-60 s$ & $7 \%$ & $4 \%$ & $6 \%$ & \multirow{2}{*}{$8 \%$} \\
\hline & $60-80 s$ & $12 \%$ & $9 \%$ & $10 \%$ & \\
\hline \multirow{2}{*}{$\begin{array}{c}\text { Dimensional } \\
\text { lifetime reduction }\end{array}$} & $0-50 \mathrm{~m}^{2} / \mathrm{s}$ & $40 \%(29 \%-40 \%)$ & $29 \%$ & $22 \%$ & \multirow{4}{*}{$\begin{array}{c}21 \% \\
(21 \%-31 \%)\end{array}$} \\
\hline & $50-100 \mathrm{~m}^{2} / \mathrm{s}$ & $20 \%(14 \%-22 \%)$ & $15 \%$ & $4 \%$ & \\
\hline \multirow{2}{*}{$\begin{array}{c}\text { Normalized } \\
\text { lifetime reduction }\end{array}$} & $0.1-0.2$ & $34 \%(25 \%-37 \%)$ & $25 \%$ & $19 \%$ & \\
\hline & $0.2-0.3$ & $23 \%(15 \%-26 \%)$ & $17 \%$ & $6 \%$ & \\
\hline
\end{tabular}

Within the weight category Heavy the B767-300ER was the most frequently captured aircraft type. The B767300ER falls into category C "Lower Heavy" of the RECAT-EU scheme and contributes about $1.6 \%$ of all landings at Vienna airport. Figure 13 left depicts wake vortex measurements of 20 approaches of B767-300ER aircraft with plate lines corresponding to 54 vortex evolutions and 19 approaches without plate lines corresponding to 52 vortex evolutions. The two plots on the left consider wake transport and decay in the $\pm 50 \mathrm{~m}$ safety corridor. Figure 13 right shows the subset of cases where the last measurement is situated within the safety corridor and is based on 26 vortex evolutions with and 30 vortex evolutions without plate lines.
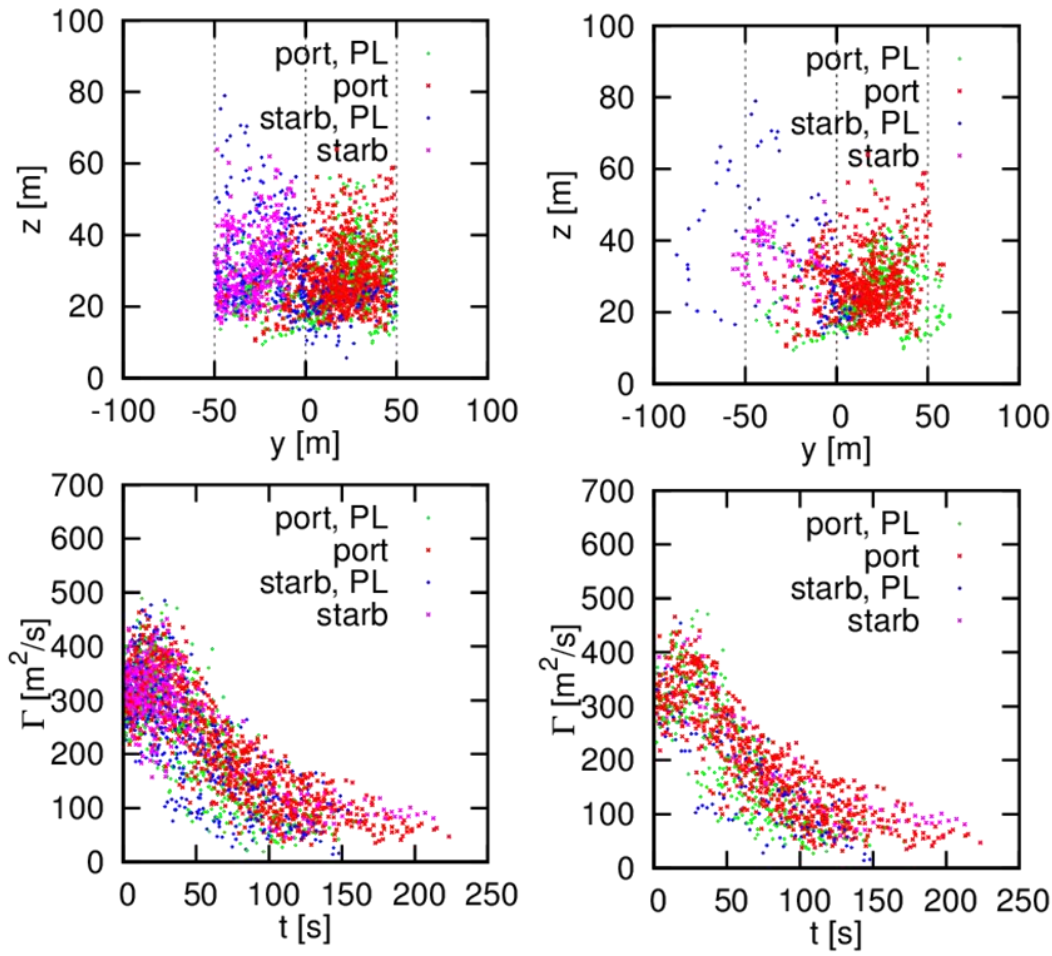

Figure 13 Measurements of B767 wake vortices for headwinds lower than $2 \mathrm{~m} / \mathrm{s}$. Vortices residing within the $\pm 50 \mathrm{~m}$ safety corridor (left) and complete vortex evolutions where the last vortex observation lies within the safety corridor (right).

Due to the lower amount of data in the scatter plots one may readily identify that the circulation decays faster with erected plate lines. Considering the data counts and percentile plots in Figure 14 this becomes even more evident. Within the ICAO separation relevant vortex age bins from $100 \mathrm{~s}$ to $140 \mathrm{~s}$ the circulation is reduced on average by 53\% (Figure 14 top right and Table 4). Also the vortex-age-binned circulation decay displayed in Figure 14 bottom progresses faster with the plates and the maximum lifetimes are noticeably reduced.

15

American Institute of Aeronautics and Astronautics 
The $\lambda$-shape of the circulation-binned percentiles is clearly visible. The individual percentile curves diverge into the $\lambda$ legs at a circulation level of $250-300 \mathrm{~m}^{2} / \mathrm{s}$ for the dimensional plot (bottom right) and at 0.5 to $0.7 \Gamma_{0}$ for the normalized representation (bottom left). The reduced vortex lifetimes listed in Table 4 vary between 17\% and 30\% for the different criteria. The average of the lifetime reductions of the long-lived vortices (circulations bins $0-100$ $\mathrm{m}^{2} / \mathrm{s}$ and $0.1-0.3$ ) amounts to $25 \%$. The confidence interval of the medians resides between $20 \%$ and $39 \%$.
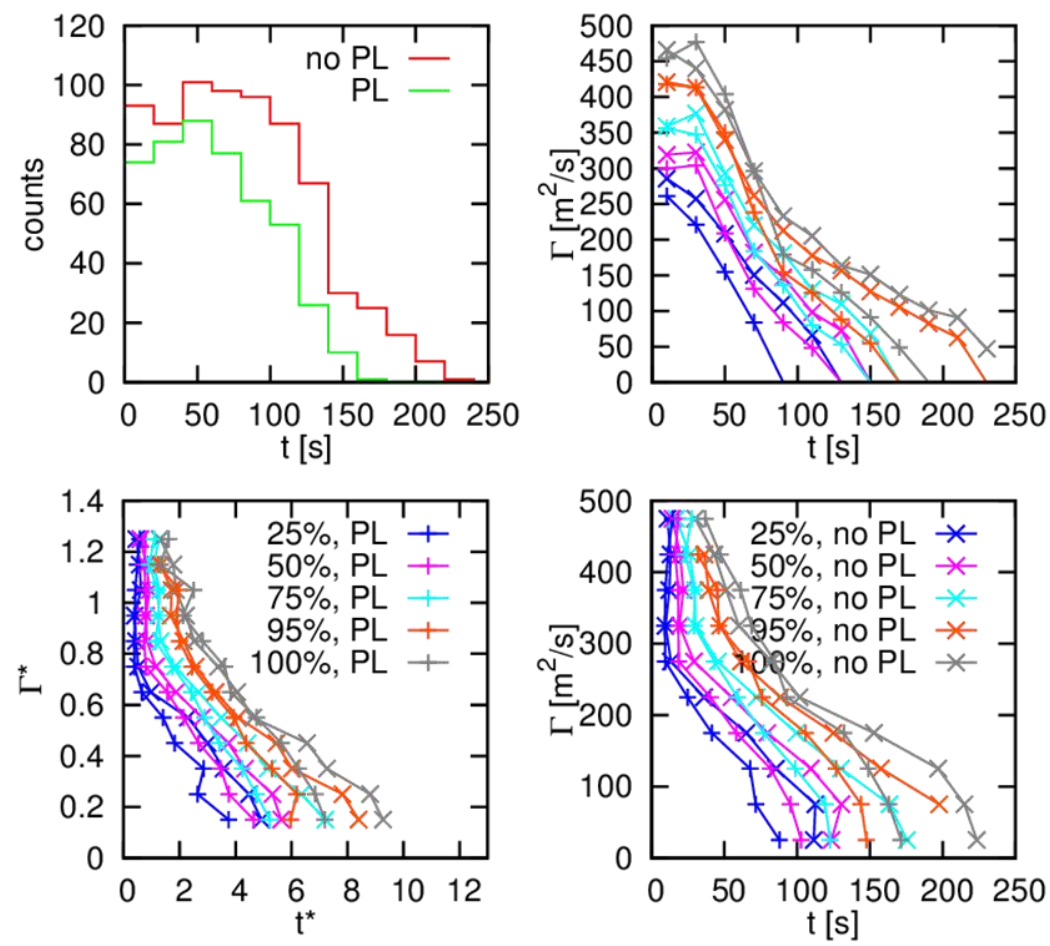

Figure 14 B767 vortex measurement counts (top left), vortex-age-binned circulation percentiles (top right), and circulation-binned normalized (bottom left) and dimensional (bottom right) vortex age percentiles in the $\pm 50 \mathrm{~m}$ safety corridor for headwinds lower than $2 \mathrm{~m} / \mathrm{s}$.

Table 4 Reductions of B767 wake vortex circulations and lifetimes with the $\pm 50 \mathrm{~m}$ safety corridor for headwinds lower than $2 \mathrm{~m} / \mathrm{s}$.

\begin{tabular}{cccccc}
\hline \hline & Bins & $50^{\text {th }}$ percentile & $75^{\text {th }}$ percentile & $95^{\text {th }}$ percentile & average \\
\hline Dimensional & $100-120 \mathrm{~s}$ & $51 \%$ & $39 \%$ & $29 \%$ & \multirow{2}{*}{$53 \%$} \\
circulation reduction & $120-140 \mathrm{~s}$ & $100 \%$ & $52 \%$ & $44 \%$ & \\
Dimensional & $0-50 \mathrm{~m}^{2} / \mathrm{s}$ & $17 \%(10 \%-44 \%)$ & $30 \%$ & - & \\
lifetime reduction & $50-100 \mathrm{~m}^{2} / \mathrm{s}$ & $27 \%(26 \%-39 \%)$ & $27 \%$ & $27 \%$ & $25 \%$ \\
Normalized & $0.1-0.2$ & $17 \%(17 \%-33 \%)$ & $28 \%$ & $29 \%$ & $(20 \%-39 \%)$ \\
lifetime reduction & $0.2-0.3$ & $29 \%(28 \%-41 \%)$ & $25 \%$ & $21 \%$ & \\
\hline \hline
\end{tabular}

The B777-200 is with 30 landings the most frequent representative of the RECAT-EU scheme B "Upper Heavy" within the Vienna data base (1.2\% traffic share). In detail the B777-200 data sample contains 14 approaches with plate lines corresponding to 41 vortex evolutions and 16 approaches without plates represented by 38 vortex evolutions. Figure 11 features quite impressive maximum vortex lifetimes. The longest-lived B777-200 vortex reaches a lifetime of $281 \mathrm{~s}$ within the safety corridor while the port vortex of the same flight reaches an age of $315 \mathrm{~s}$ outside the safety corridor in lidar plane 5. Figure 11 right shows the sparsely populated subset of cases where the last measurement is situated within the safety corridor for the balanced number of 8 vortex evolutions with and without plate lines, respectively. 

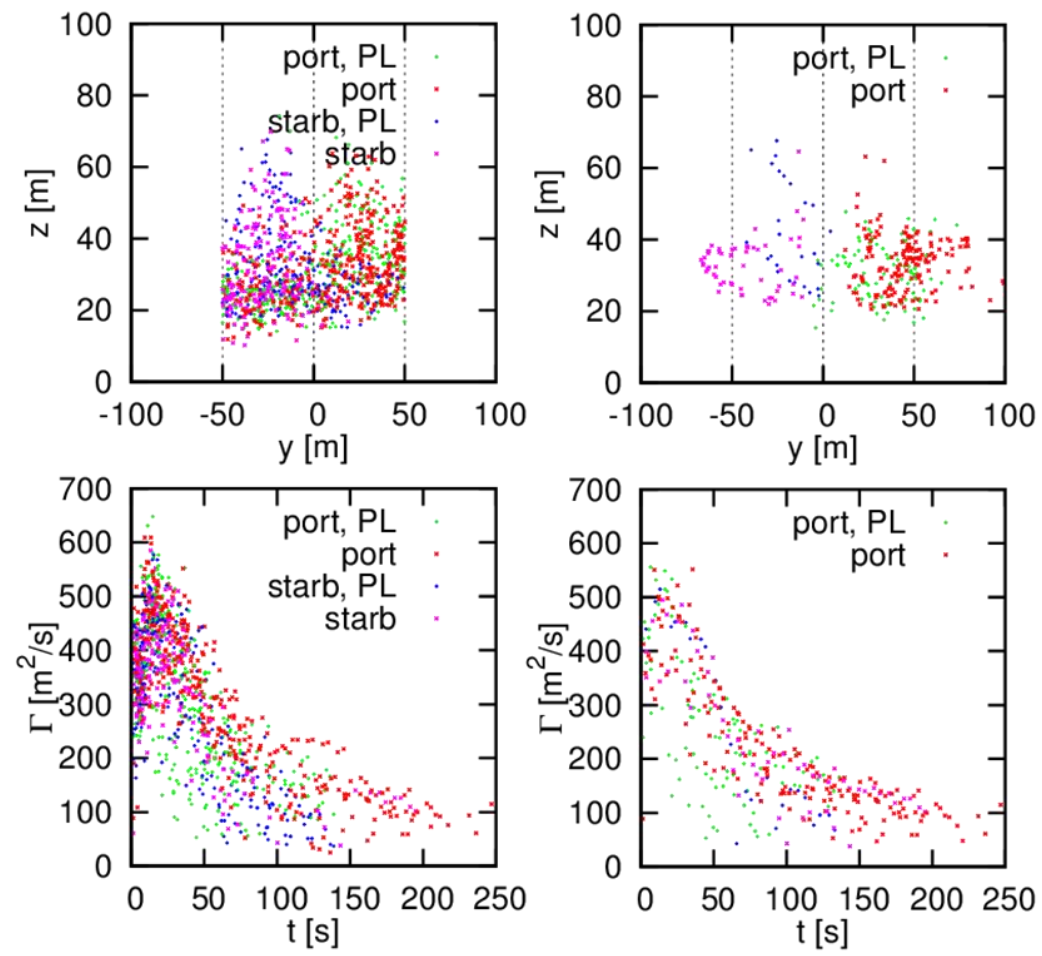

Figure 15 Measurements of B777-200 wake vortices for headwinds lower than $2 \mathrm{~m} / \mathrm{s}$. Vortices residing within the $\pm 50 \mathrm{~m}$ safety corridor (left) and complete vortex evolutions where the last vortex observation lies within the safety corridor (right).
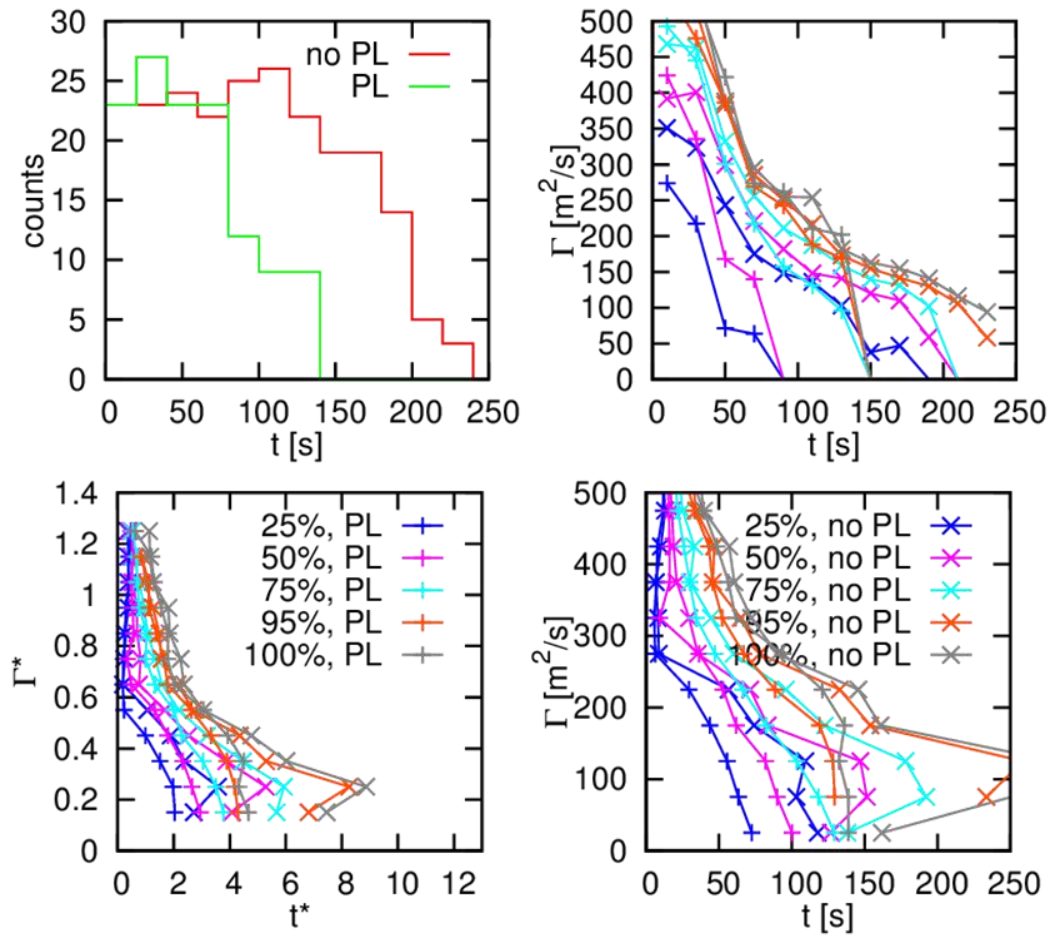

Figure 16 B777-200 vortex measurement counts (top left), vortex-age-binned circulation percentiles (top right), and circulation-binned normalized (bottom left) and dimensional (bottom right) vortex age percentiles in the $\pm 50 \mathrm{~m}$ safety corridor for headwinds lower than $2 \mathrm{~m} / \mathrm{s}$. 
Figure 16 and Fehler! Ungültiger Eigenverweis auf Textmarke. feature impressive wake vortex mitigation rates with respect to the vortex counts and the circulation and lifetime reduction potentials. The lowest dimensional circulation bin doesn't contain enough data to pass the Wilcoxon test. As a consequence the $95 \%$ confidence interval of the median is extremely wide and even extends to negative values. Further, the $75^{\text {th }}$ percentile features an unrealistically low lifetime reduction potential. However, on average the circulation reduction of $48 \%$ and the lifetime reduction of 35\% are quite high and backed up by the Wilcoxon test. So the circulation bin $200 \mathrm{~m}^{2} / \mathrm{s}-250$ $\mathrm{m}^{2} / \mathrm{s}$, where the $\lambda$-legs are splitting up, features already a p-value of $10^{-5}$. The limited availability of data for the B777-200 is also reflected in the widest interval for the average median lifetime reductions ranging from $14 \%$ to $53 \%$.

Table 5 Reductions of B777-200 wake vortex circulations and lifetimes with the $\pm 50 \mathrm{~m}$ safety corridor for headwinds lower than $2 \mathrm{~m} / \mathrm{s}$.

\begin{tabular}{cccccc}
\hline \hline & Bins & $50^{\text {th }}$ percentile & $75^{\text {th }}$ percentile & $95^{\text {th }}$ percentile & average \\
\hline Dimensional & $100-120 \mathrm{~s}$ & $100 \%$ & $30 \%$ & $13 \%$ & $48 \%$ \\
circulation reduction & $120-140 \mathrm{~s}$ & $100 \%$ & $40 \%$ & $2 \%$ & \\
Dimensional & $0-50 \mathrm{~m}^{2} / \mathrm{s}$ & $21 \%(-9 \%-46 \%)$ & $7 \%$ & - & \\
lifetime reduction & $50-100 \mathrm{~m}^{2} / \mathrm{s}$ & $41 \%(24 \%-55 \%)$ & $39 \%$ & $45 \%$ & $35 \%$ \\
Normalized & $0.1-0.2$ & $28 \%(12 \%-58 \%)$ & $33 \%$ & $37 \%$ \\
lifetime reduction & $0.2-0.3$ & $50 \%(30 \%-54 \%)$ & $41 \%$ & $50 \%$ & $(14 \%-53 \%)$ \\
\hline \hline
\end{tabular}

\section{E. Turbulence Effects}

Turbulence intensity is retrieved from the measurements of the microwave scintillometer combined with the large aperture scintillometer observing in the near infrared spectral region. The combined system includes two transmitters and two receivers situated in positions A and B shown in Figure 3. The signal variances of the two systems are translated into the respective structure parameters $C_{n}^{2}$ in order to define two different turbulence regimes. The relative deviations of the two $C_{n}^{2}$ values from their respective medians are used to assign the individual overflights to either the low turbulence or the high turbulence regime.

Again the analysis employs the safety corridor criteria and uses only cases with headwinds lower than $2 \mathrm{~m} / \mathrm{s}$. Scintillometer data is only available for a part of the lidar measurements such that the number of the analyzed measurements is slightly reduced compared to the previous analyses. The low-turbulence regime consists of 169 vortex evolutions with and without plate lines in the safety corridor, respectively, (see leftmost plots in Figure 17), whereas 45 (37) cases with (without) plate lines are considered where the last vortex is situated within the safety corridor (center-left plots in Figure 17). The right side of Figure 17 depicts the corresponding cases for the highturbulence regime with 116 cases with and without plate lines in the safety corridor (center-right), respectively, and 33 (40) cases with (without) plate lines in the rightmost plots showing vortices over their entire lifetime.

Apparently, higher environmental turbulence accelerates wake vortex decay with and without plate lines. In the high-turbulence regime most vortices have decayed before vortex ages of $100 \mathrm{~s}$ whereas in the low-turbulence regime similar degrees of decay are not reached before $150 \mathrm{~s}$. The $50^{\text {th }}$ percentile features average vortex lifetime reductions driven by increased turbulence without plates by $26 \%$ in the two lowest circulation bins. This is in the same order of magnitude of the lifetime reductions achieved by the plate lines (see Table 6). Two high-turbulence cases with lifetimes beyond $150 \mathrm{~s}$ are standing out that have a strong impact on the $95^{\text {th }}$ and $100^{\text {th }}$ percentiles shown in Figure 18 right. In both cases the structure parameters are close to the threshold separating low and high turbulence overflights. So these two cases may somewhat distort the overall figures listed in Table 6.

In summary, the relative circulation and lifetime reduction potential of plate lines appears to be similar in low and high-turbulence conditions. Because stronger turbulence directly reduces wake vortex duration and strength substantially, the highest potential for the mitigation of wake vortex risks lies within low wind (see section V.C.) and low turbulence conditions where without plate lines the longest-lived vortices occur and are leading to the highest encounter risks. 
low turbulence
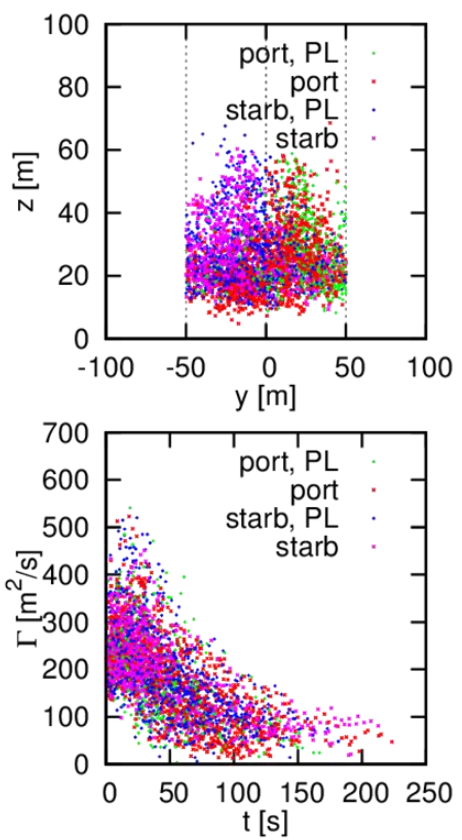
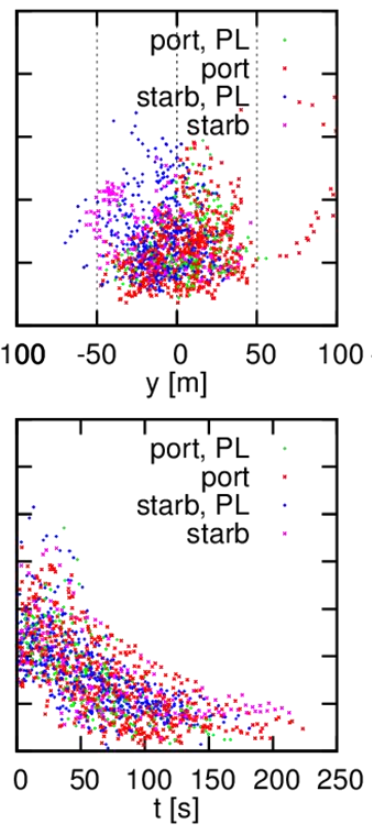

high turbulence
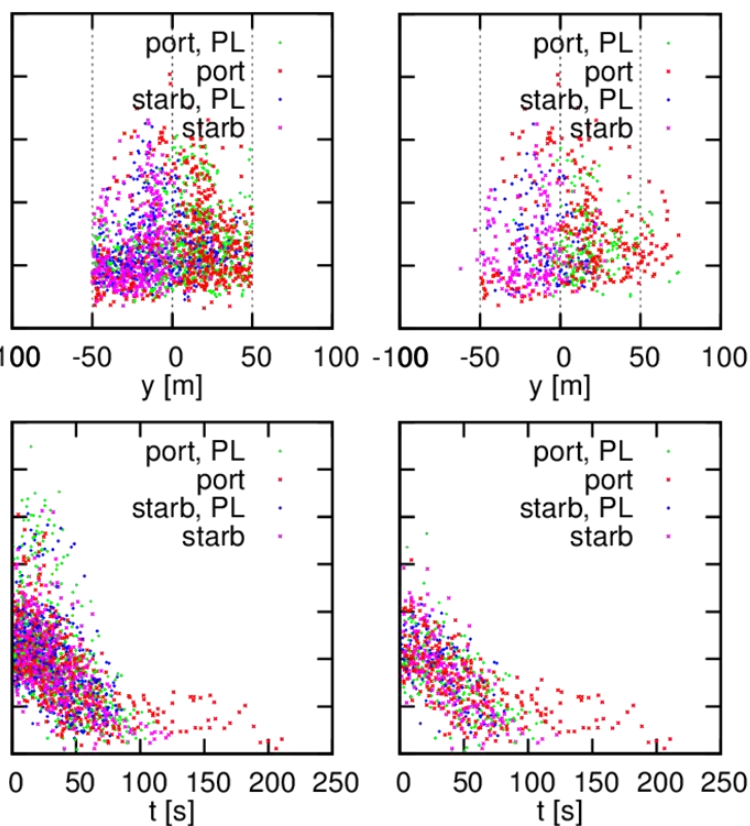

Figure 17 Wake vortex measurements within different turbulence regimes for headwinds lower than $2 \mathrm{~m} / \mathrm{s}$.

low turbulence
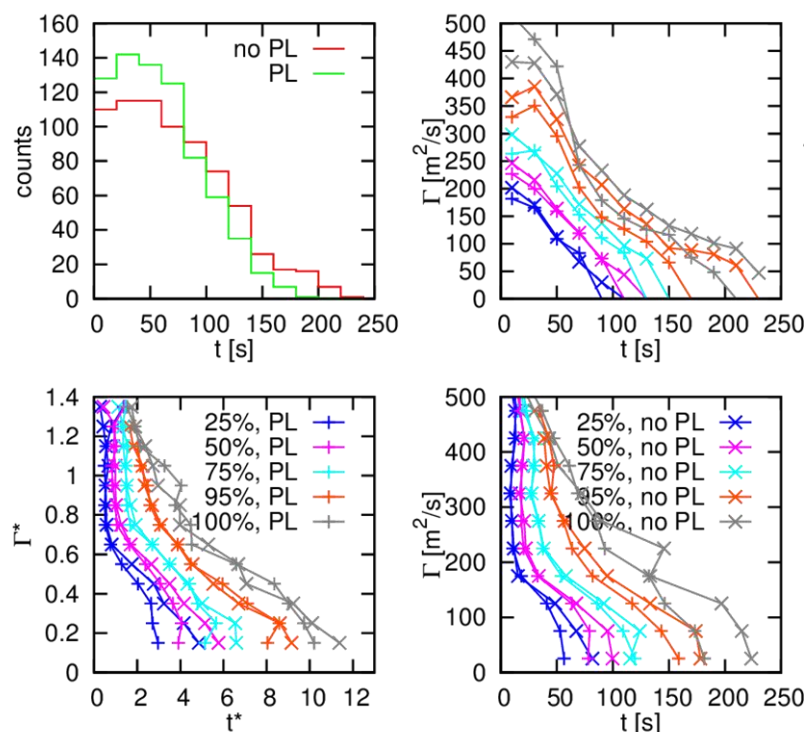

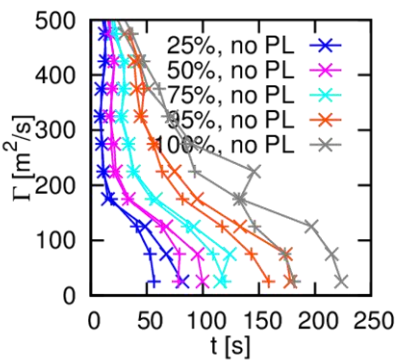

$\mathrm{t}[\mathrm{s}]$ high turbulence
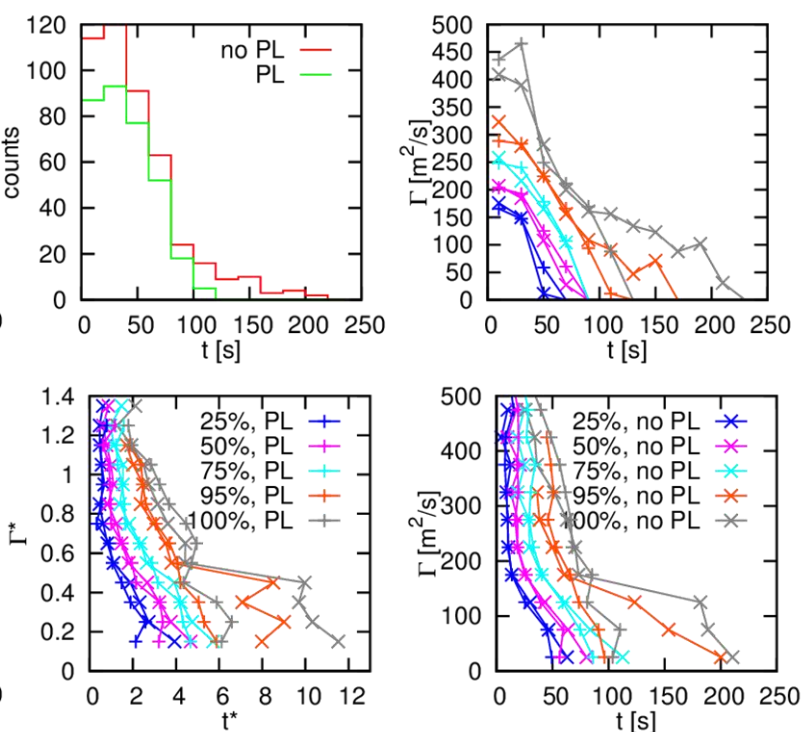

Figure 18 Wake vortex measurement counts, vortex-age-binned circulation percentiles, and circulation-binned vortex age percentiles in the $\pm 50 \mathrm{~m}$ safety corridor for headwinds lower than $2 \mathrm{~m} / \mathrm{s}$ within different turbulence regimes. 
Table 6 Reductions of wake vortex circulations and lifetimes with the $\pm 50 \mathrm{~m}$ safety corridor for headwinds lower than $2 \mathrm{~m} / \mathrm{s}$ within two turbulence regimes.

\begin{tabular}{lcccccc}
\hline \hline & & Bins & $50^{\text {th }}$ percentile & $75^{\text {th }}$ percentile & $95^{\text {th }}$ percentile & average \\
\hline & Dimensional & $100-120 \mathrm{~s}$ & $100 \%$ & $13 \%$ & $23 \%$ & \\
& circulation reduction & $120-140 \mathrm{~s}$ & - & $100 \%$ & $23 \%$ & $52 \%$ \\
Low & Dimensional & $0-50 \mathrm{~m}^{2} / \mathrm{s}$ & $21 \%(5 \%-29 \%)$ & $-6 \%$ & $16 \%$ & \\
turb. & lifetime reduction & $50-100 \mathrm{~m}^{2} / \mathrm{s}$ & $22 \%(12 \%-26 \%)$ & $16 \%$ & $22 \%$ & \\
& Normalized & $0.1-0.2$ & $31 \%(23 \%-38 \%)$ & $21 \%$ & $16 \%$ & $(14 \%-32 \%)$ \\
& lifetime reduction & $0.2-0.3$ & $23 \%(15 \%-33 \%)$ & $21 \%$ & $2 \%$ & \\
\hline & Dimensional & $100-120 \mathrm{~s}$ & - & - & $88 \%$ & \\
& circulation reduction & $120-140 \mathrm{~s}$ & - & - & $100 \%$ & \\
High & Dimensional & $0-50 \mathrm{~m}^{2} / \mathrm{s}$ & $30 \%(5 \%-45 \%)$ & $23 \%$ & $52 \%$ & \\
turb. & lifetime reduction & $50-100 \mathrm{~m}^{2} / \mathrm{s}$ & $7 \%(-1 \%-21 \%)$ & $11 \%$ & $41 \%$ & \\
& Normalized & $0.1-0.2$ & $32 \%(10 \%-48 \%)$ & $18 \%$ & $26 \%$ & $(3 \%-36 \%)$ \\
& lifetime reduction & $0.2-0.3$ & $10 \%(-2 \%-29 \%)$ & $9 \%$ & $41 \%$ & \\
\hline \hline
\end{tabular}

\section{F. Plate Line Coverage}

One pertinent question is how many plate lines are required to mitigate wake turbulence risks within the complete area where wake vortices may rebound to the flight path and cause vortex encounters. This question is related to the propagation speed and outreach of the disturbances triggered by the plates. To answer these questions measurements were conducted during which only plate line 1 was upright, which is the one situated closer to the runway next to the localizer (see Figure 3). The favorable effects achieved with a single plate line are compared to those achieved with two plate lines. Because lidar measurements in position L2 directly above plate line 1 should not differ between both setups with one or two plate lines, only measurements taken in lidar positions L3 to L5 are included in this analysis (see Figure 3). Because the number of heavy aircraft measured with a single plate line is very low, the analysis is restricted to vortices generated by A320 aircraft.

The reference case with both plate lines depicted on the left side of Figure 19 and Figure 20 consists of 163 vortex evolutions with plate lines and 164 vortex evolutions without plates. The right parts of these two figures are based on 76 cases with plate line 1 and 77 cases without plates. Apart from the different number of measurements the results with one and two plate lines appear very similar. This is also reflected by the similar lifetime reductions listed in Table 7 which differ on average only by $2 \%$. This $2 \%$ variation is significantly smaller than the median ranges estimated by the Wilcoxon test for $95 \%$ confidence levels (numbers in brackets).

Overall, the presented data plus the consideration of additional analyses with varying sample sizes partly also including heavy aircraft suggest that a single plate line might be a little less effective than two lines but should be sufficient to trigger most of the desired favorable plate line effects. 

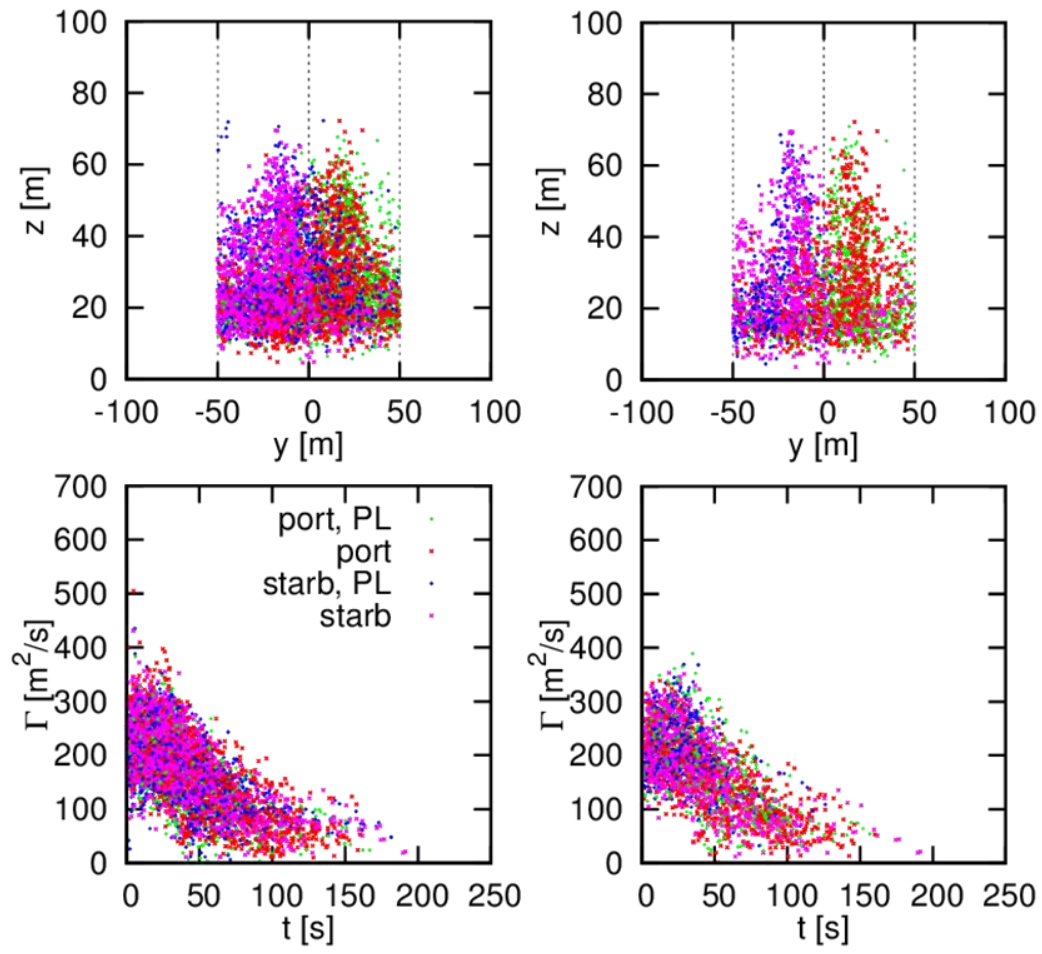

Figure 19 Measurements of A320 wake vortices in $\pm 50 \mathrm{~m}$ safety corridor for headwinds lower than $2 \mathrm{~m} / \mathrm{s}$ with both plate lines (left) and only plate line 1 (right).
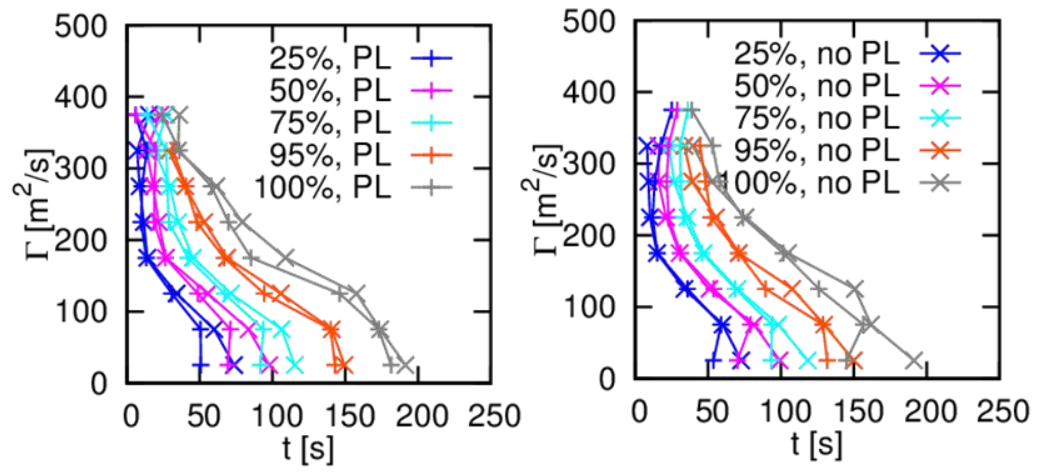

Figure 20 A320 vortex age percentiles in the $\pm 50 \mathrm{~m}$ safety corridor for headwinds lower than $2 \mathrm{~m} / \mathrm{s}$ with both plate lines (left) and only plate line 1 (right).

Table 7 Reductions of A320 wake vortex lifetimes with both plate lines (top) and only a single plate line (bottom).

\begin{tabular}{ccccccc}
\hline \hline & & Bins & $50^{\text {th }}$ percentile & $75^{\text {th }}$ percentile & $95^{\text {th }}$ percentile & average \\
\hline Both plate & Dimensional & $0-50 \mathrm{~m}^{2} / \mathrm{s}$ & $29 \%(17 \%-35 \%)$ & $21 \%$ & $4 \%$ & \multirow{2}{*}{$\begin{array}{c}14 \% \\
\text { lines }\end{array}$} \\
lifetime reduction & $50-100 \mathrm{~m}^{2} / \mathrm{s}$ & $15 \%(6 \%-17 \%)$ & $12 \%$ & $1 \%$ & $(11 \%-26 \%)$ \\
Single & Dimensional & $0-50 \mathrm{~m}^{2} / \mathrm{s}$ & $29 \%(4 \%-37 \%)$ & $21 \%$ & $12 \%$ & \multirow{2}{*}{\}} \\
plate line & lifetime reduction & $50-100 \mathrm{~m}^{2} / \mathrm{s}$ & $3 \%(-4 \%-10 \%)$ & $4 \%$ & $1 \%$ & $(0-24 \%)$ \\
\hline \hline
\end{tabular}




\section{Conclusion}

At Vienna International Airport a measurement campaign has been accomplished from 6 May 2019 until 28 November 2019 in order to assess the potential of plate lines to mitigate wake turbulence risks during final approach. The wake decay enhancing method is passive, low-cost, robust, and safe. Wake vortices generated by landing aircraft induce secondary vortices at the plates' surfaces that approach the primary vortices and trigger premature wake vortex decay. The installation of the plates increases flight safety and reduces the number of wake vortex encounters and the thereby provoked go-arounds. Furthermore, the concept may enable capacity gains via synergies with other measures for the optimization of aircraft separations.

Two plate lines were erected below the glide path to runway 16 and wake vortex behavior was gathered employing three wind lidars complemented by a suite of meteorological instrumentation. Wake vortex behavior of about 9,500 approaches has been collected in five different lidar measurement planes covering the area in which wake vortices may rebound to the approach path corridor. From these over 1000 wake vortex evolutions with and without plate lines have been processed and used for the current analysis.

The developed evaluation strategy focuses on safety aspects:

- The conducted analysis employs an aircraft safety corridor extending $\pm 50 \mathrm{~m}$ from the runway centerline. Vortices transported out of that safety area are neglected for the analysis. The sensitivity to the width of the safety corridor turned out to be weak. A larger safety corridor slightly decreases the plate line effectiveness, because additional plates ahead of a vortex strengthen the plate-induced vortex decay.

- The data indicates that vortex decay is correlated with headwind speed. Because strong headwinds support the transport of end effects (occurring during touchdown and accelerating vortex decay) into the measurement planes, cases with headwinds stronger than $2 \mathrm{~m} / \mathrm{s}$ are excluded for the further analysis. Encounter frequencies and go-around rates are highest at weak headwinds or even tailwinds.

- The analysis combines data of all the available lidar measurement planes, because the accelerated vortex decay should be effective all along the stretch where rebounding vortices may reach up to the flight corridor. The data samples collected in the five individual lidar planes appeared too small to support conclusive results.

- To enable the comparison of wake vortices generated by different aircraft types, the data characterizing vortex decay is partly normalized employing aircraft parameters retrieved from the Mode-S protocol and aircraft masses provided by Austrian Airlines.

- The analysis evaluates the lifetime reduction of long-lived wake vortices. For vortices at a progressed state of decay (circulation bins below $100 \mathrm{~m}^{2} / \mathrm{s}$ and normalized circulation bins below $30 \%$ of the initial circulation) the median, the $75^{\text {th }}$ percentile, and the $95^{\text {th }}$ percentile of the vortex lifetime are considered. For reasons of clarity, the 12 values resulting from four circulation bins with three percentiles are averaged to a single percentage of lifetime reduction.

- The circulation reduction is considered for vortex lifetimes around $60 \mathrm{~s}$ for Medium vortex generating aircraft and around $120 \mathrm{~s}$ for Heavies. Again the relative circulation reductions achieved for the three selected percentiles are averaged into a single value.

- Finally, the Wilcoxon rank-sum test is used to assess whether the differences of the lifetime distributions with and without plate lines are statistically significant or not. Further, margins of uncertainty of the median lifetime reductions are determined with $95 \%$ confidences.

Following all the above listed criteria, the lifetime of the long-lived vortices in the safety corridor is reduced on average by $24 \%$ for 420 landings comprising medium, heavy, and super weight class aircraft. For this 456 vortex evolution measurements with plate lines and 472 vortex evolutions without plates have been considered. The median lifetime reductions according to the Wilcoxon test range from $23 \%$ to $32 \%$. The average circulation reduction around vortex ages of $120 \mathrm{~s}$, corresponding to the ICAO separation for Mediums behind Heavies, amounts to 66\%.

It is found that increased environmental turbulence reduces median wake vortex lifetimes by the same order of magnitude as the lifetime reductions achieved by the plate lines. The circulation and lifetime reduction potentials of plate lines appear to be similar in low and high-turbulence conditions. So the highest potential for the mitigation of wake vortex risks lies within low wind and low turbulence conditions where without plate lines the longest-lived vortices occur and are leading to the highest encounter risks.

The question how many plate lines are required to mitigate wake turbulence risks within the complete area where wake vortices may rebound to the flight path and cause vortex encounters is investigated by comparing 
measurements with one and two plate lines. The data suggests that a single plate line might be a little less effective than two lines but should be sufficient to trigger most of the desired favorable effects. Obviously, the propagation speed and outreach of the disturbances triggered by the plates are quite high.

The mitigation potential of wake turbulence risks via plate lines is considered for the most frequent aircraft at Vienna airport for three different weight categories of the RECAT-EU scheme. As representative of the ICAO weight category Medium and category D "Upper Medium" of the RECAT-EU scheme, 348 approaches of the A320 aircraft corresponding to 337 vortex evolutions with plate lines and 203 approaches without plates have been assessed. For this smaller aircraft type circulation and lifetime reductions are less pronounced than for larger aircraft. The average circulation reduction for the A320 is assessed for vortex ages around $60 \mathrm{~s}$ corresponding to radar separation and amounts to $8 \%$. The lifetime reductions of the long-lived vortices average to $21 \%$, whereas the medians of the lifetime reductions with $95 \%$ confidence vary between $21 \%$ and $31 \%$.

Within the weight category Heavy and category C "Lower Heavy" of the RECAT-EU scheme the B767-300ER was the most frequently captured aircraft type with 39 approaches. Based on 54 vortex evolutions with and 52 vortex evolutions without plate lines the reduced vortex lifetimes average to $25 \%$ with a confidence interval of the median of $20 \%$ to $39 \%$. Within the ICAO separation relevant vortex age bins centered around $120 \mathrm{~s}$ the circulation is reduced on average by $53 \%$.

The B777-200 with 30 landings is the most frequent representative of the RECAT-EU scheme B "Upper Heavy". For this aircraft type 41 vortex evolutions with plate lines are compared to 38 vortex evolutions without plates. The longest-lived B777-200 vortex reaches a lifetime of $315 \mathrm{~s}$. The average circulation reduction of $48 \%$ and the average lifetime reduction of $35 \%$ are quite high and backed up by the Wilcoxon test notwithstanding the relatively small sample size.

Despite the relatively high scatter of individual lidar circulation measurements, the presented statistics appear sound. So the circulation percentiles used to estimate wake vortex lifetime are featuring $\lambda$ shapes. Initially, the percentiles for the cases with and without plate lines proceed almost identically, demonstrating that random fluctuations stemming from varying environmental conditions and stochastic vortex behavior as well as measurement uncertainties have smoothed out. Then the curves split up into the $\lambda$-legs when the plate induced decay comes into effect. This instant coincides with the time when the Wilcoxon test attests the transition from statistically similar vortex age distributions to distinctly different distributions with high confidences transitioning from p-values like 0.7 to $10^{-10}$.

In summary, it can be concluded that the plate lines substantially reduce both the lifetime of long-lived vortices as well as their circulation at vortex ages relevant for aircraft separations in a safety corridor along the final approach path. The lifetime reductions increase with aircraft size from 21\% for the A320 to 35\% for the B777-200. For the two investigated Heavy aircraft types the mean circulation reduction amounts to 50\% assuming a $120 \mathrm{~s}$ separation to following Medium weight class aircraft.

Next steps will comprise the assessment of achievable reductions of aircraft separation minima by applying the rationale developed for the definition of RECAT-EU separations to the collected lidar data for all three RECAT phases. Further, a technical plate design for permanent deployment at an airport will be elaborated with the goal to obtain the approval from the authorities for its installation.

\section{Acknowledgments}

This project has received funding within the framework of the SESAR Joint Undertaking "Increased Runway and Airport Throughput" project (PJ.02 EARTH) within the European Union's Horizon 2020 research and innovation programme under grant agreement No 731781 and the German Aerospace Research Center (DLR) project "Wetteroptimierter Luftverkehr". The provision of landing masses by W. Wurzinger from Austrian Airlines is greatly acknowledged.

\section{References}

\footnotetext{
${ }^{1}$ Hallock, J.N., Holzäpfel, F., “A Review of Recent Wake Vortex Research for Increasing Airport Capacity”, Progress in Aerospace Sciences, Vol. 98, 2018, pp. 27-36, https://doi.org/10.1016/j.paerosci.2018.03.003.

2 Eurocontrol, "European Aviation in 2040 - Challenges of growth 2018", 2 Oct. 2018, https://www.eurocontrol.int/publication/challengesgrowth-2018.

${ }^{3}$ ICAO, “GUIDANCE ON A380-800 WAKE VORTEX ASPECTS”, TEC/OPS/SEP - 08-0294.SLG, 8 July 2008

${ }^{4}$ Crouch, J.D., Czech, M.J., "Comparative wake wake-turbulence assessments and findings for the B747-8", 4th WakeNet3-Europe workshop, Langen, Germany, 2012, http://www.wakent.eu/index.php?id=185.
} 
${ }^{5}$ Duffy, K., Deluca, A., “One year of time-based separation at London's Heathrow airport”, WakeNet-USA, Port Authority of NY \& NJ, New York City, 2016.

${ }^{6}$ SAFO 14007, "Safety Alert for Operators, Federal Aviation Administration," 2014, http://www.faa.gov/other_visit/aviation industry/airline operators/airline_safety/safo.

${ }^{7}$ EUROCONTROL, 2016, http://www.eurocontrol.int/news/recat-eu-now-use-paris-charles-de-gaulle.

${ }^{8}$ Schumann, U. (ed.), From Fundamental Research to Operational Application, Research Topics in Aerospace, Atmospheric Physics Background - Methods - Trends, Springer, Berlin Heidelberg, 2012, p. 225, http://dx.doi.org/10.1007/978-3-642-30183-4.

${ }^{9}$ Critchley, J., Foot, P., “UK CAA Wake Vortex Database: Analysis of Incidents Reported Between 1982 and 1990", Civil Aviation Authority, CAA Paper 91, 1991.

${ }^{10}$ WakeNet3-Europe, “Aircraft Wake Vortex State-of-the-Art \& Research Needs", compiled by F. Holzäpfel et al., issued by A. Reinke, C. Schwarz, 2015, 201 pages, doi.org/10.17874/BFAEB7154B0.

11 Holzäpfel, F., Steen, M., "Aircraft Wake-Vortex Evolution in Ground Proximity: Analysis and Parameterization," AIAA Journal, Vol. 45, 2007, pp. 218-227.

12 Bricteux, L., Duponcheel, M., De Visscher,I., Winckelmans, G., "LES investigation of the transport and decay of various-strengths wake vortices in ground effect and subjected to a turbulent crosswind," Physics of Fluids, Vol. 28, 2016, pp. 065105-1-065105-28, https://doi.org/10.1063/1.4954150.

${ }^{13}$ Frank Holzäpfel, Nikola Tchipev, Anton Stephan, "Wind Impact on Single Vortices and Counterrotating Vortex Pairs in Ground Proximity", Flow, Turbulence and Combustion, Vol. 97, 2016, pp. 829-848, http://dx.doi.org/10.1007/s10494-016-9729-2.

$\checkmark{ }^{14}$ Holzäpfel, F., "Analysis of potential wake vortex encounters at a major European airport," Aircraft Engineering and Aerospace Technology, Vol. 89, 2017, pp. 634-643, http://dx.doi.org/10.1108/AEAT-01-2017-0043.

$\checkmark{ }^{15}$ Holzäpfel, F., Stephan, A., Heel, T., Körner, S., "Enhanced Wake Vortex Decay in Ground Proximity Triggered by Plate Lines," Aircraft Engineering and Aerospace Technology, Vol. 88, 2016, doi: 10.1108/AEAT-02-2015-0045.

${ }^{16}$ Stephan, A., Holzäpfel, F., Misaka, T., "Aircraft Wake-Vortex Decay in Ground Proximity - Physical Mechanisms and Artificial Enhancement," Journal of Aircraft, Vol. 50, 2013, DOI:10.2514/1.C032179, pp. 1250 - 1260.

${ }^{17}$ Stephan, A., Holzäpfel, F., Misaka, T., Geisler, R., Konrath, R., "Enhancement of aircraft wake vortex decay in ground proximity," $C E A S$ Aeronautical Journal, Vol. 5, 2014, pp. 109-125, DOI 10.1007/s13272-013-0094-8.

${ }^{18}$ Stephan, A., Schrall, J., Holzäpfel, F., "Numerical Optimization of Plate-Line Design for Enhanced Wake-Vortex Decay," Journal of Aircraft, Vol. 54, 2017, pp. 995-1010, http://dx.doi.org/10.2514/1.C033973.

${ }^{19}$ Vechtel, D., Stephan, A., Holzäpfel, F., "How Plate Lines Influence the Hazard Perception of Pilots During Wake Encounters ", Journal of Aircraft, Vol. 57, 2020, pp. 360-367, https://doi.org/10.2514/1.C035689.

${ }^{20}$ Holzäpfel, F., Stephan, A., Rotshteyn, G., "Plate Lines Reduce Lifetime of Wake Vortices During Final Approach to Vienna Airport," AIAA Paper 2020-0050, AIAA SciTech Forum 2020, Orlando, FL, 18 pages.

- ${ }^{21}$ Wang, C.H.J., Zhao, D., Schlüter, J., Holzäpfel, F., Stephan, A., "LES study on the shape effect of ground obstacles on wake vortex dissipation", Aerospace Science and Technology, Vol. 63, 2017, pp. 245-258, http://dx.doi.org/10.1016/j.ast.2016.12.032.

${ }^{22}$ Wang, C.H.J., Zhao, D., Schlüter, J., Stephan, A., Holzäpfel, F., "Computational Investigation of Varying Plate-Line Geometry and Placement on Wake Vortex Dissipation”, Journal of Aircraft, Vol. 55, 2018, pp. 133-144, https://doi.org/10.2514/1.C034346.

${ }^{23}$ Geise, R., Neubauer, B., Zimmer, G., "Scaled Measurements of Multipath Propagation and Navigations Systems - a Practical Example for ILS," 2019 Joint International Symposium on Electromagnetic Compatibility, Sapporo and Asia-Pacific International Symposium on Electromagnetic Compatibility (EMC Sapporo/APEMC), 2019, http://dx.doi.org/10.23919/EMCTokyo.2019.8893769.

${ }^{24}$ International Civil Aviation Organization, “Autodrome Design Manual”, Part 6 Frangibility, Doc 9157, AN/901, 2006.

${ }^{25}$ Stephan, A., Holzäpfel, F., Misaka, T., "Hybrid simulation of wake-vortex evolution during landing on flat terrain and with plate line", International Journal of Heat and Fluid Flow, Vol. 49, 2014, pp. 18-27, http://dx.doi.org/10.1016/j.ijheatfluidflow.2014.05.004.

${ }^{26}$ Smalikho, I.N. , Banakh, V.A., Holzäpfel, F., Rahm, S., "Method of radial velocities for the estimation of aircraft wake vortex parameters from data measured by coherent Doppler lidar," Optics Express, Vol. 23, pp. A1194-A1207, 2015, http://dx.doi.org/10.1364/OE.23.0A1194.

${ }^{27}$ Gurke, T., Lafferton, H., "The Development of the Wake Vortices Warning System for Frankfurt Airport: Theory and Implementation," Air Traffic Control Quarterly, Vol. 5, 1997.

${ }^{28}$ BADA, User Manual for the Base of Aircraft Data (BADA) Revision 3.11, EEC Technical/Scientific Report No. 13/04/16-01, EUROCONTROL, May 2013, 109 pages. http://upcommons.upc.edu/bitstream/handle/2099.1/24342/AnnexI.pdf?sequence=2

${ }^{29}$ Wilcoxon, F., "Individual comparisons by ranking methods," Biometrics Bulletin, Vol. 1, No. 6, $1945, \quad$ pp. 80-83. http://dx.doi.org/10.2307/3001968

${ }^{30} \mathrm{R}$ Core Team, "R: A language and environment for statistical computing," R Foundation for Statistical Computing, Vienna, Austria, 2019, https://www.R-project.org/. 
This article has been cited by:

1. Frank Holzäpfel, Lukas Strauss, Carsten Schwarz. 2021. Assessment of dynamic pairwise wake vortex separations for approach and landing at Vienna airport. Aerospace Science and Tecbnology 112, 106618. [Crossref] 